Article

\title{
Small RNA Sequencing Analysis of miRNA Expression Reveals Novel Insihts into Root Formation under Root Restriction Cultivation in Grapevine (Vitis vinifera L.)
}

\author{
Hui Li ${ }^{1}$, Zhen Gao ${ }^{2}$, Muhammad Salman Zahid ${ }^{1}$, Dongmei Li ${ }^{1}$, Hafiz Umer Javed ${ }^{1}$, Lei Wang ${ }^{1}$, \\ Shiren Song ${ }^{1}$, Liping Zhao ${ }^{1}$, Wenping $\mathrm{Xu}{ }^{1}$, Caixi Zhang ${ }^{1}$, Chao Ma ${ }^{1, *}$ and Shiping Wang ${ }^{1,3, *}$ \\ 1 Department of Plant Science, School of Agriculture and Biology, Shanghai Jiao Tong University, \\ Shanghai 200240, China; oldhorseli@sjtu.edu.cn (H.L.); salmanzahid2482@hotmail.com (M.S.Z.); \\ LDM651011@sjtu.edu.cn (D.L.); qaziumer@sjtu.edu.cn (H.U.J.); leiwang2016@sjtu.edu.cn (L.W.); \\ sr.song@sjtu.edu.cn (S.S.); lpzhao07@sjtu.edu.cn (L.Z.); wp-xu@sjtu.edu.cn (W.X.); acaizh@sjtu.edu.cn (C.Z.) \\ 2 State Key Laboratory of Crop Biology, College of Horticulture Science and Engineering, \\ Shandong Agricultural University, Tai'an 271018, China; gaoz89@126.com \\ 3 Institute of Agro-food Science and Technology/Key Laboratory of Agro-products Processing Technology of \\ Shandong, Shandong Academy of Agricultural Sciences, Jinan 250100, China \\ * Correspondence: chaoma2015@sjtu.edu.cn (C.M.); fruit@sjtu.edu.cn (S.W.)
}

Received: 2 April 2020; Accepted: 12 May 2020; Published: 15 May 2020

\begin{abstract}
Root restriction cultivation (RRC) can influence plant root architecture, but its root phenotypic changes and molecular mechanisms are still unknown. In this study, phenotype observations of grapevine root under RRC and control cultivation (nRC) at 12 time points were conducted, and the root phenotype showed an increase of adventitious and lateral root numbers and root tip degeneration after RRC cultivation from 70 days after planting (DAP). The 70 and 125 DAP sampling of two different cultivations, named nR70, RR70, nR125, and RR125, were selected for small RNA sequencing. A total of 153 known miRNAs and 119 predicted novel miRNAs were obtained. Furthermore, BLAST was used to predict the novel miRNAs with miRBase databases using the default parameters; 96 of the 119 predicted novel miRNAs were similar to other species, and the remaining 23 grapevine-specific novel miRNAs were obtained. There were 26, 33, 26, and 32 miRNAs that were differentially expressed in different comparison groups (RR70 vs. nR70, RR125 vs. nR125, nR125 vs. nR70 and RR125 vs. RR70). Target genes prediction of differentially expressed miRNAs was annotated on a variety of biological processes, and 24 participated in root development. Moreover, multiple miRNAs were found to jointly regulate lateral root development under root restriction conditions. The miRNA expression pattern comparison between RRC and $\mathrm{nRC}$ may provide a framework for the future analysis of miRNAs associated with root development in grapevine.
\end{abstract}

Keywords: microRNA; Vitis vinifera; root restriction; root architecture; high-throughput sequencing

\section{Introduction}

Roots, as an important organ of perennial fruit trees, not only have a mechanical support function for growth, but play a key role in the process of absorbing water and mineral nutrients from the soil $[1,2]$. Normally, plant roots occur in the radicle of seeds, and root formation can be divided into primary roots, lateral roots, and adventitious roots [3]. The primary root is the first root grown by the plant, usually as the main root; when the main root grows to a certain length, lateral roots emerge from the proximal end of the main root, and continue to emit secondary, tertiary, or multi-level lateral roots; adventitious roots 
refer to roots emanating from the stems and leaves, which can also emit multi-level lateral roots [4]. Grapevine is a dicotyledonous plant that can be propagated through seeds. During seed propagation, the root system is a taproot system, that is, there are obvious main roots and lateral roots. At the same time, grapevine can also be asexually propagated through cuttings. The root system of the cuttings is formed not by a main root, but by adventitious roots emanating from the stems. The genotypes of offspring obtained by asexual reproduction are the same, and are widely used in scientific research $[5,6]$.

In the early 1990s, researchers were inspired by the practice of garden plant potting and began to explore the cultivation method of limiting the root system of plants, which was called RRC. RRC refers to a cultivation technique that uses physical or ecological materials to control the root system of a plant within a certain volume, and to control the growth space of the root system to regulate the above-ground vegetative and reproductive growth [7]. RRC can significantly inhibit the shoot perimeter, branch length, and leaf area, while improving fruit coloring, increasing the content of pigments such as anthocyanin and carotenoid, increasing the soluble sugar content within the pulp, and reducing the tartaric acid content in grapevine berries [8,9]. These effects may due to the influence of RRC on the absorption of nitrogen and phosphorus in grapevine [10,11]. Our previous studies, in which root morphology observations were conducted, revealed that the root structure was significantly altered under RRC. The dry weight of roots $(0.2 \mathrm{~cm}<$ thickness $<0.5 \mathrm{~cm})$ and fiber roots $(<0.2 \mathrm{~cm})$ increased significantly. However, these studies lacked the systematic observation of the whole process of root development. Moreover, there has been little research the molecular mechanisms of grapevine root development, especially under RRC.

Non-coding RNA (ncRNA), which does not encode proteins, is a type of RNA that is widely present in many organisms [12,13], and plays an important role in regulating plant growth and development. Among them, microRNA (miRNA) is a class of endogenous small non-coding RNA with a length of 19-25 nucleotides [14]. MiRNA has been widely reported in various plants and animals and plays a crucial role in post-transcriptional regulation or transcriptional suppression of genes [15]. Plant miRNA not only affects plant resistance to biotic and abiotic stresses, but directly participates in plant growth and development. There have been many miRNAs also reported to influence root development $[16,17]$. For example, overexpression of ath-miR164 reduced lateral root numbers in Arabidopsis [18], and miR160 maintained proper auxin homeostasis and root tip development by regulating ARF17 in Arabidopsis [19,20]. MiR390 influenced lateral root development by regulating the expression of $A R F 2, A R F 3$, and ARF4 through the secondary siRNA produced by the TAS3 (trans-acting siRNA 3) gene [21]. Except for these conserved miRNAs, it was reported that some novel miRNAs also participated in root development. For example, miR2111 was found to regulate root node development by long distance transportation [22].

In recent years, high throughput sequencing has been widely used in grapevine to analyze miRNA functions in biotic stress, such as aster yellow phytoplasma infection [23], and abiotic stress, such as cold stress [24]. Moreover, a microRNA expression atlas of grapevine was analyzed, including 70 small RNA libraries containing many tissues [25,26]. In addition, Chitarra et al. integrated a novel miRNA database "miRVIT" inferred from published small RNA libraries that were not uploaded into the miRBase database [27]. However, the miRNA derived from the grapevine root system had less statistical sequencing data. In this study, we analyzed the root phenotype and the expression profile of miRNAs during grapevine root development under both normal and root restriction cultivations, which will provide a framework for the future analysis of miRNAs associated with root development in grapevine.

\section{Results}

\subsection{Phenotype Variations of Grapevine cv. Muscat Hamburg after Root Restriction Cultivation}

The shoot lengths of one-year-old Vitis vinifera L. cv. Muscat Hamburg specimens were measured from 60 to 125 days after planting (DAP) under two different cultivations. The results showed that 
the shoot length of nRC grew faster and reached about $5.5 \mathrm{~m}$, one meter longer than that of RRC (Supplementary Materials Figure S1A). The base diameters of the new shoots in nRC were always higher than those of RRC (Supplementary Materials Figure S1B). The number and length of the secondary shoots were measured between two pruning times, and the length was divided into four grades, denoted I-IV (Supplementary Materials Figure S1C,D). The results showed that the number of secondary shoots in $\mathrm{nRC}$ was 18-20 per tree, which was almost two fold greater than the 8-11 per tree of RRCs (Supplementary Materials Figure S1E). Moreover, the length and diameter of the secondary shoots in $\mathrm{nRC}$ were significantly higher than those in RRC (Supplementary Materials Figure S1F,G).

Root phenotype photographs were taken at 12 sampling time points $(10,20,30,40,50,60,70,80$, 90, 100, 110, and 125 days after planting) from April 24 to August 18, and the $\mathrm{nRC}$ and RRC were recorded as $\mathrm{nR}+\mathrm{DAP}$ (Figure S2) and RR + DAP, respectively (Supplementary Materials Figure S3). The two cultivations showed similar root formation orders including absorbing roots, secondary lateral roots, and new adventitious roots. Finally, the old roots degenerated, and new adventitious roots developed into the main root system. However, after the 70 DAP sampling, the root morphology of the two cultivations changed significantly.

Compared with the $\mathrm{nRC}$, a large number of new adventitious roots with thinner diameters emerged in RRC. Adventitious roots occurred in clusters in both cultivations (Figure 1A,B). The number and diameter of the cluster adventitious roots were analyzed at 70 DAP. The results showed that the number increased significantly after RRC to eight adventitious roots per cluster, which was about twice that of the nRC (four per cluster) (Figure 1C). Furthermore, the diameter of each of the cluster adventitious roots in RRC was about $0.15 \mathrm{~cm}$, which was significantly lower than the nRC (about $0.22 \mathrm{~cm}$ ) (Figure 1D). The root tip was intact in nRC (Figure 1E), while the root tip growth was defective in RRC, which led to a large number of clustered roots that emerged from the degenerated root tips (Figure 1F). The lateral roots were mainly distributed in the upper part of the roots in nRC (Figure 1G), but in RRC, the lateral roots were densely distributed on the whole roots at 125 DAP (Figure 1H). Occasionally, root tip defects appeared in $\mathrm{nRC}$, and a large number of cluster roots were also emitted from the defective parts (Figure 1I). However, the same type of roots emitted more lateral roots, including secondary or multiple lateral roots, under the RRC (Figure 1J). Root update speed was accelerated after RRC. In the same root, while new roots continued to occur at the lower end, the upper roots began to brown, decay, and disappear (Supplementary Materials Figure S4). 
A

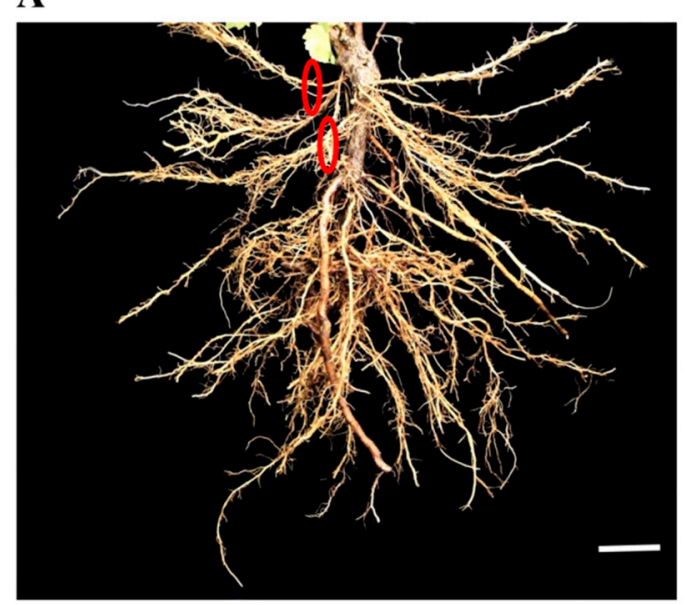

C

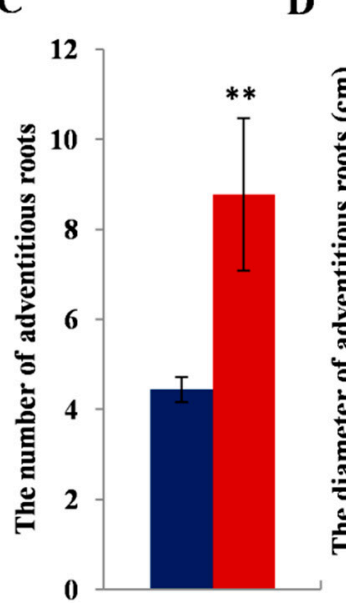

D

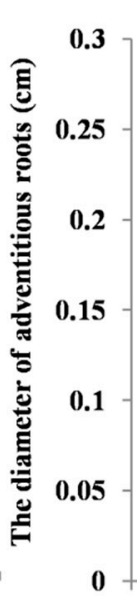

B

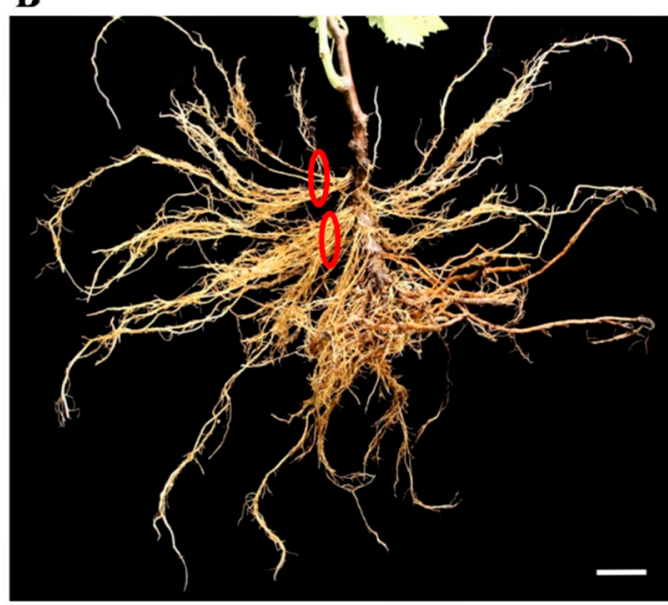

$\mathbf{E}$

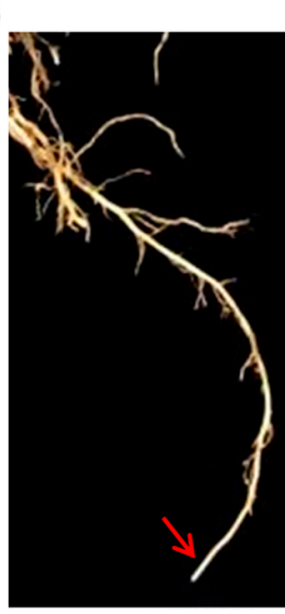

F

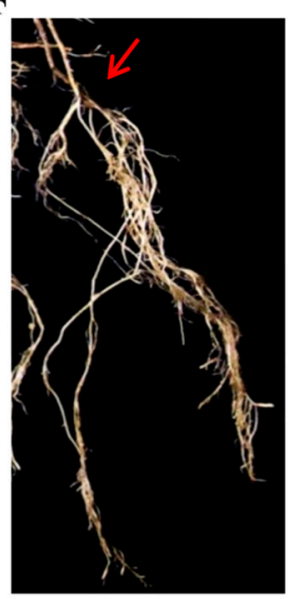

G

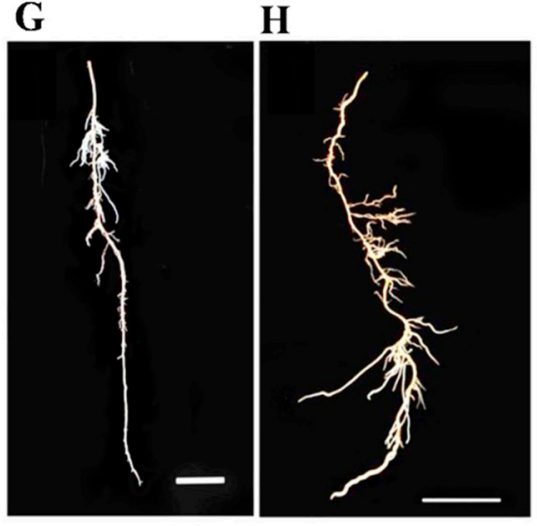

I

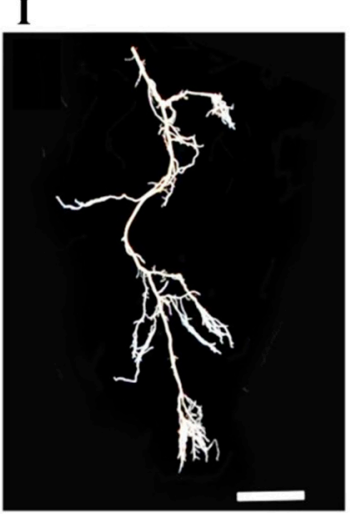

$\mathbf{J}$

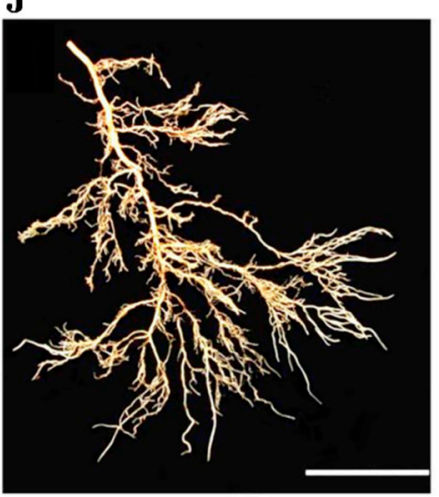

Figure 1. Root phenotype variations of one-year-old grapevine (Vitis vinifera L. cv. Muscat Hamburg) after root restriction cultivation. The new adventitious root development in control (A) and root restriction cultivation (RRC) (B); red circles represent adventitious roots that occurred in clusters. (C,D) The number and diameter of adventitious roots in two cultivation models; error bars indicate the mean $\pm \mathrm{SD}(\mathrm{n}>10)$ with biological replicates, and values $\left.{ }^{* *}\right)$ are statistically significant from the control cultivation based on Student's t test $(P<0.01)$. The root tip in control cultivation $(\mathbf{E})$ and RRC $(\mathbf{F})$; red arrows represent the initial root tip position; $(\mathbf{G}, \mathbf{I})$ the root morphology in different root positions in control cultivation; $(\mathbf{H}, \mathbf{J})$ the root morphology in the same root position in RRC. Scale bar $=5 \mathrm{~cm}$. 


\subsection{Sequencing Statistics in Different Grapevine Samples}

The continuous phenotypic observations of the grapevine root system revealed that there were significant differences between the 70 DAP samplings in RRC and nRC. Next, the 70 and 125 DAP root samples were selected for small RNA sequencing. These four root samples were named as nR70, nR125, RR70, and RR125, and each sample had three replicates, recorded as A, B, and C, respectively. A total of 214,439,588 raw reads were obtained and 168,741,687 clean reads were finally obtained after the quality control steps. The clean reads of each library were between 11.29 and $15.60 \mathrm{M}$ (Table 1). The copy number of FastUniq clean reads ranging from one to ten were more than $96.5 \%$, among which single and double copies accounted for $69.72 \%$ and $15.24 \%$, respectively, reaching a total of $85 \%$ (Supplementary Materials Figure S5).

Table 1. The statistical information of miRNA in high throughput sequencing.

\begin{tabular}{ccccccc}
\hline Sample & Raw Reads & $\begin{array}{c}\text { Reads Trimmed } \\
\text { Length }\end{array}$ & $\begin{array}{c}\text { Reads } \\
\text { Trimmed_Q20 }\end{array}$ & $\begin{array}{c}\text { Reads } \\
\text { Trimmed_N }\end{array}$ & $\begin{array}{c}\text { Clean Reads } \\
\text { Clean Reads } \\
\text { Uniq }\end{array}$ \\
\hline nR70A & $17,440,227$ & $13,828,456$ & $13,805,784$ & $13,791,775$ & $13,791,775$ & $2,789,195$ \\
nR70B & $16,785,530$ & $12,712,507$ & $12,694,953$ & $12,682,543$ & $12,682,543$ & $2,424,073$ \\
nR70C & $16,705,937$ & $12,741,508$ & $12,719,806$ & $12,706,889$ & $12,706,889$ & $2,449,057$ \\
RR70A & $19,603,314$ & $16,149,312$ & $16,130,501$ & $16,129,531$ & $16,129,531$ & $3,896,588$ \\
RR70B & $16,454,204$ & $13,692,986$ & $13,676,330$ & $13,662,899$ & $13,662,899$ & $3,129,670$ \\
RR70C & $14,509,081$ & $11,845,746$ & $11,840,120$ & $11,839,622$ & $11,839,622$ & $2,768,680$ \\
nR125A & $17,921,125$ & $14,407,233$ & $14,391,593$ & $14,377,909$ & $14,377,909$ & $3,583,714$ \\
nR125B & $18,875,954$ & $14,074,226$ & $14,050,637$ & $14,036,500$ & $14,036,500$ & $3,639,479$ \\
nR125C & $17,047,738$ & $12,015,900$ & $11,997,503$ & $11,985,776$ & $11,985,776$ & $3,224,084$ \\
RR125A & $19,152,378$ & $15,408,723$ & $15,401,439$ & $15,400,735$ & $15,400,735$ & $3,942,147$ \\
RR125B & $20,246,983$ & $16,368,921$ & $16,361,306$ & $16,360,589$ & $16,360,589$ & $4,167,784$ \\
RR125C & $19,697,117$ & $15,784,039$ & $15,767,917$ & $15,766,919$ & $15,766,919$ & $4,360,497$ \\
Total & $214,439,588$ & $169,029,557$ & $168,837,889$ & $168,741,687$ & $168,741,687$ & $40,374,968$ \\
\hline
\end{tabular}

\subsection{Identification of Known and Novel Grapevine miRNAs}

After a series of miRNAs prediction analyses, a total of 153 known grapevine miRNAs, and 119 novel miRNAs (named by chromosome random number) were obtained. The length distribution results showed that the known miRNAs were distributed between 19 and 24 nts (nucleotides), of which more than $60 \%$ were $21 \mathrm{nts}$ miRNAs (Figure 2A). The length of novel miRNA ranged from 18 to $25 \mathrm{nts}$, of which the first peak was $23 \mathrm{nts}$, which accounted for more than $35 \%$, and the proportion was slightly higher in RRC; the second length peak was $21 \mathrm{nts}$, which accounted for about $25 \%$ (Figure 2B). The miRBase database recorded a total of 48 known miRNA families in grapevine, and 45 of them were detected in this study except miR828, miR2950, and miR3628 families. Only 30 miRNA members from 13 known grapevine miRNA families were not detected. The predicted novel miRNAs were searched using the BLAST miRVIT database and 18 of them were perfectly matched. Among them, nine were similar to known miRNA families in grapevine: Un_39994, 6_13658, 19_26046, 19_26048, and 19_25033 were similar to vvi-miR477a; 1_21167 was similar to vvi-miR482; 14_36566 and 17_1792 were similar to vvi-miR3627-5p; and 14_37516 was similar to vvi-miR3633b-3p (Supplementary Materials Table S1). 
A

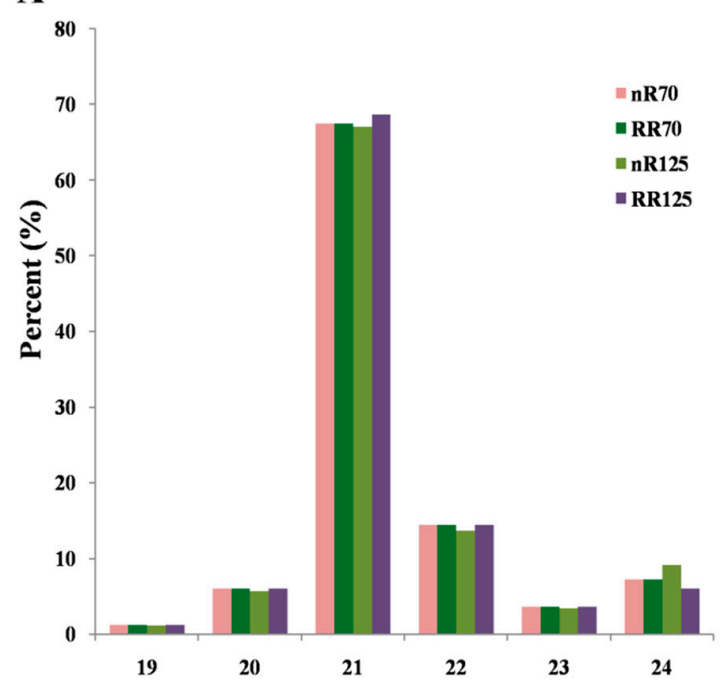

B

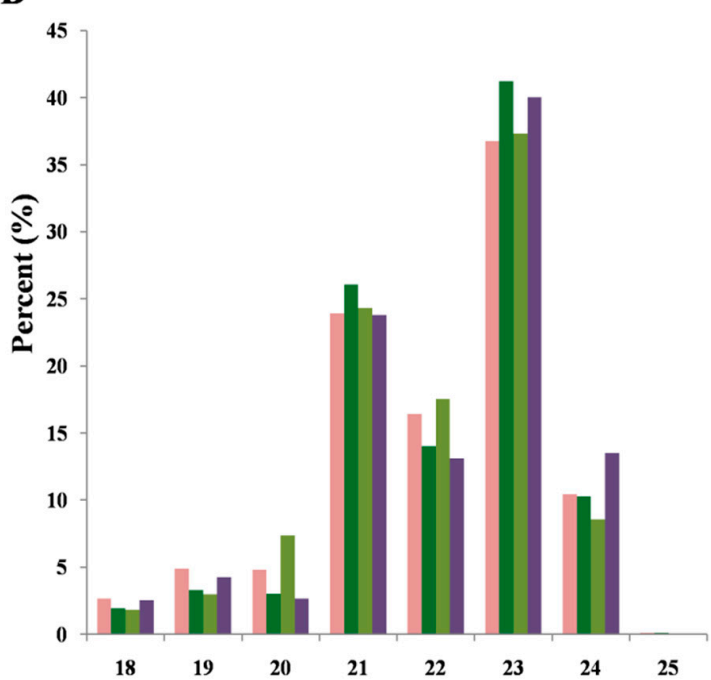

Figure 2. The length distribution percentage of the known (A) and novel (B) miRNAs in four root sequencing samples. nR70, RR70, nR125 and RR125 represented the 70 days after planting, simplified DAP and 125 DAP sampling time points under control and root restriction cultivation.

To obtain the detailed information of the predicted grapevine novel miRNAs, they were aligned with the mature miRNAs database of miRBase using the default parameters. Fourteen conserved grapevine miRNA family members were obtained (Figure 3). Seven of the 14 conserved miRNAs were also detected in the miRVIT database, but they were much better matched in the miRBase than in the miRVIT database. Among them, five novel miRNAs annotated in miRVIT, which were similar to vvi-miR477a, matched better in the miRBase database to ppt-miR477f (Physcomitrella patens). Meanwhile, 1_21167 and 14_37516 matched better with mtr-miR482-3p (Medicago truncatula) and gma-miR482a -3p (Glycine max), respectively. The remaining seven novel miRNAs were 6_12672, 17_2431,11_7793, 9_19848, 15_8904, 2_4979, and 9_20339, which were similar to csi-miR159b-5p (Citrus sinensis), mes-miR159a-5p (Manihot esculenta), vvi-miR396b, csi-miR156f-3p, ath-miR162a-3p (Arabidopsis thaliana), seu-miR319 (Salicornia europaea), and osa-miR396e-3p (Oryza sativa), respectively (Table 2). In addition, there were 82 novel miRNAs aligned to miRNA families that had been reported in other species but not in grapevine (Supplementary Materials Table S3). The miRNAs 5_32700 and ghr-miR827a (Gossypium hirsutum), 14_36566 and vca-miR391-5p (Vrieseacarinata), and 14_37655 and mes-miR1446 each had an alignment score of more than 90 points, and mature sequences had high sequence homology with other species (Supplementary Materials Table S2). The remaining 23 novel miRNAs that did not match either database were considered as grapevine-specific novel miRNAs (Supplementary Materials Table S3). 

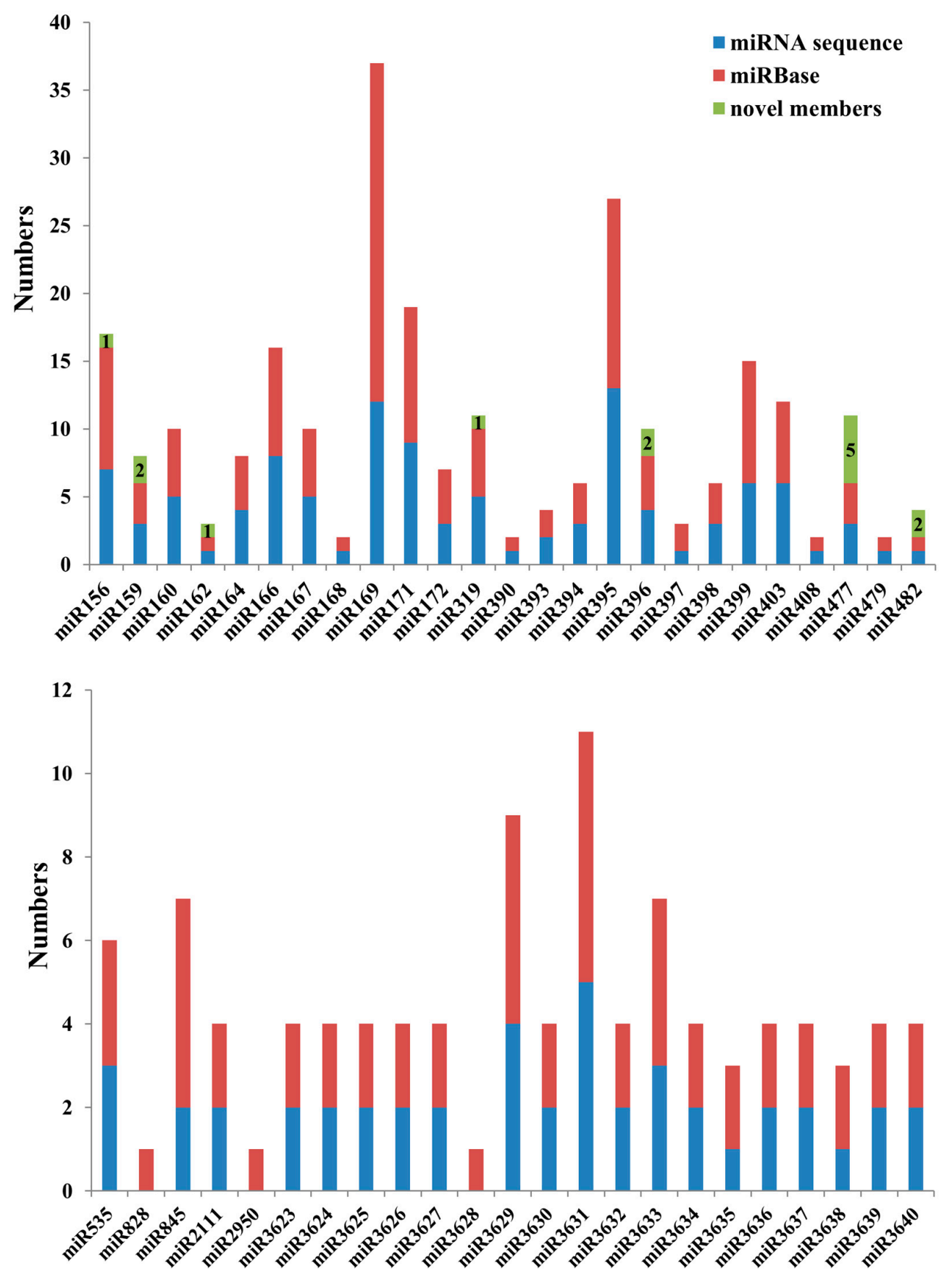

Figure 3. The number of known miRNA family members in grapevine obtained from the miRNA sequence, and the predicted novel miRNA further determined using BLAST with the miRBase. 
Table 2. Predicted new known miRNA member information after alignment with mature sequence in miRBase with default parameters.

\begin{tabular}{|c|c|c|c|c|c|c|c|c|c|}
\hline Provisional ID & Mature Sequence & Length & Loc_miRNA & $\begin{array}{l}\text { miRBase } \\
\text { Alignment }\end{array}$ & $\begin{array}{c}\text { Query } \\
\text { Alignment }\end{array}$ & $\begin{array}{c}\text { Subject } \\
\text { Alignment }\end{array}$ & Strand & Score & Evalue \\
\hline 6_12672 & AGCUGCCGACUCAUUCAUUCA & 21 & chr6:9137310_9137389:+ & csi-miR159b-5p & 1_21 & 1_21 & + & 105 & 0.002 \\
\hline $17 \_2431$ & AGCUGCUGACUUAUGGAUCCC & 21 & chr17:2609257_2609342:- & mes-miR159a-5p & $1 \_21$ & $1 \_21$ & + & 87 & 0.065 \\
\hline 9_19848 & CUCUCUGCUACCGUCAUUCUGC & 22 & chr9:19106874_19106939:+ & csi-miR156f-3p & 1_18 & 4_21 & + & 63 & 7 \\
\hline $1 \overline{15} 8904$ & UCGAGAAACCUCUGCAUCA & 19 & chr15:9049770_9049811:+ & ath-miR162a-3p & $1 \_18$ & $1 \_18$ & + & 81 & 0.2 \\
\hline 2_4979 & AUUGAAUGAUGCGGGAGACA & 20 & chr2:855616_855687:- & seu-miR319 & 3_20 & 3_20 & + & 81 & 0.22 \\
\hline 11_7793 & UCCCACAGCUUUCUUGAACUU & 21 & chr11:5246803_5246886:- & vvi-miR396b & $1 \_20$ & $1 \_20$ & + & 91 & 0.03 \\
\hline 9_20339 & CUUUCUUGAACCAAUGGGUCCCAUU & 25 & chr9:5984685_5984768:- & osa-miR396e-3p & $1 \_13$ & $2 \_14$ & - & 65 & 4.3 \\
\hline 19_26046 & GUUGGAAGUCGGUGGGGGACC & 21 & chr19:18872730_18872799:- & ppt-miR477f & 1_18 & $1 \_18$ & + & 90 & 0.037 \\
\hline 19_25033 & UCCCUCAAAGGCUUCCAAUUU & 21 & chr19:18678362_18678430:+ & ppt-miR477f & 1_19 & 1_18 & + & 90 & 0.037 \\
\hline 6_13658 & CUGGAAGCCGAUGGGGGACC & 20 & chr6:19950754_19950822:- & ppt-miR477f & $1 \_20$ & $1 \_18$ & + & 90 & 0.037 \\
\hline Un_39994 & UCCCUCAAAGGCUUCCAAUUUU & 22 & chrUn:16672987_16673055:- & ppt-miR477f & 1_21 & 1_18 & + & 90 & 0.04 \\
\hline $19 \_26048$ & AAGUUGGAAGCCGGUGGGGGA & 21 & chr19:18881377_18881443:- & ppt-miR477f & 3_21 & $1 \_19$ & - & 68 & 2.5 \\
\hline 14_37516 & UUCCCAAUGCCGCCCAUUCCAA & 22 & chr14:19755490_19755563:- & gma-miR482a-3p & 1_22 & 3_24 & + & 92 & 0.027 \\
\hline 1_21167 & UCUUACCAACACCUCCCAUUCCA & 23 & chr1:3865584_3865662:+ & mtr-miR482-3p & $1 \_22$ & $1 \_21$ & + & 87 & 0.065 \\
\hline
\end{tabular}




\subsection{Differentially Expressed miRNAs (DEMs) Analysis}

Principal component analysis (PCA) showed that the distribution was relatively concentrated among biological replicates. Moreover, nR70 and RR70 samples were close and some replicates could not be completely separated, whereas nR125 and RR125 could be completely separated (Supplementary Materials Figure S6). There were 26, 33, 26 and 32 differentially expressed miRNAs (DEMs) identified in different cultivations (RR70 vs. nR70; RR125 vs. nR125) and different cultivation stages (nR125 vs. nR70; RR125 vs. RR70) respectively. Among them, both the known miRNAs and novel miRNAs showed up or down regulation (Supplementary Materials Table S4). Hierarchical clustering heat map analysis showed that different replicates of the same sample clustered together. In different cultivations, both vvi-miR3627-3p and 11_random_23 were up-regulated, while vvi-miR166a, vvi-miR482, vvi-miR2111-5p, Un_39994*, and 19_26046 showed a down-regulated expression (Figure 4A). In different cultivation stages, 5_32700* and 18_33385 were up-regulated, and the down-regulated expressed miRNAs were vvi-miR3633a-3p, 17_2431*, and 2_4979 (Figure 4B). In addition, in the later development stage of the nRC (nR125 vs. nR70), vvi-miR398a, vvi-miR3623-3p, and miR3634-3p were down-regulated, and vvi-miR167b, vvi-miR319g, 6_13658, and 14_37516 were up-regulated, which showed an opposite expression trend of that in RRC (RR125 vs. nR125) (Figure 4B).
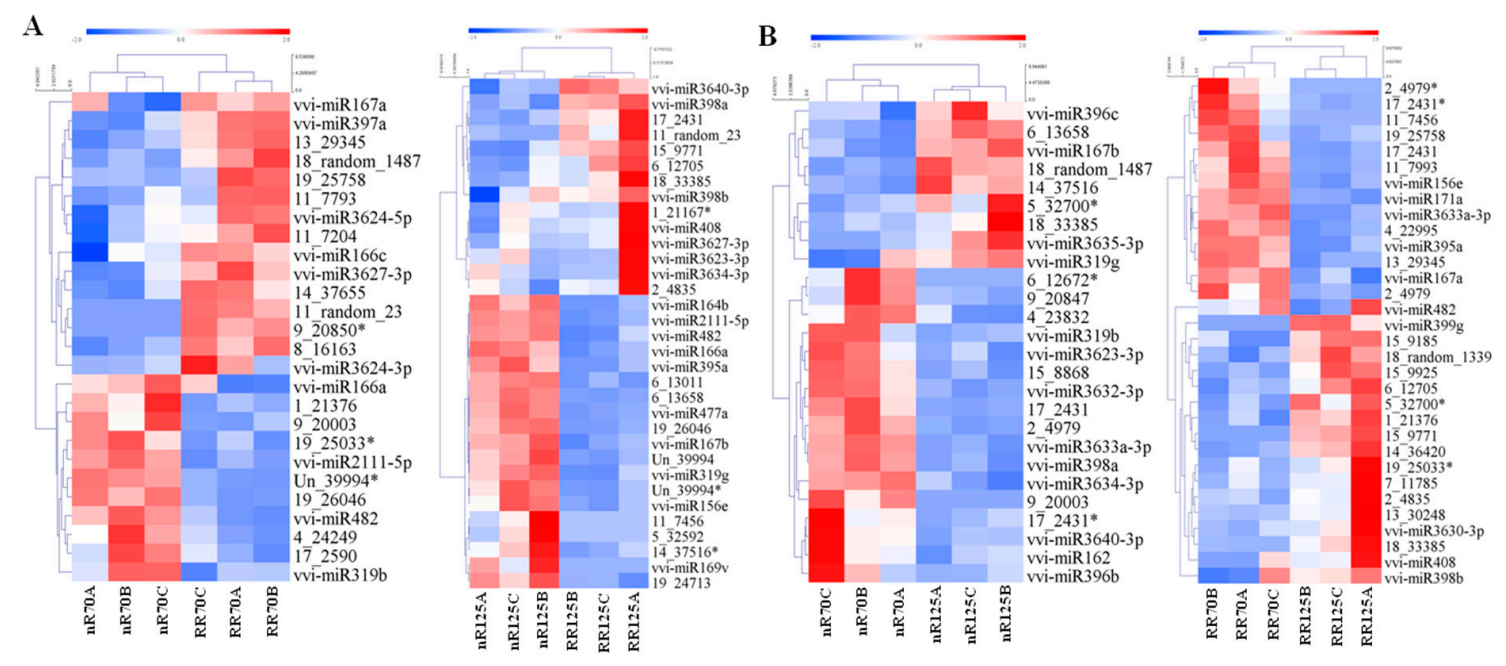

Figure 4. Hierarchical clustering heat map analysis of differentially expressed miRNAs between different cultivations (A) and different cultivation stages (B) in four root sequencing samples. nR70, RR70, nR125 and RR125 represented the 70 days after planting, simplified DAP and 125 DAP sampling time points under control and root restriction cultivation, A, B, and $C$ represent three replicates.

MiRNA expression level analysis found that known miRNA had higher transcript per million (TPM) values. Seventeen known miRNA had TPM values higher than 30, and the highest value reached was 3000 (Figure 5A). There were only 11 novel miRNAs with TPM values above 10 (Figure 5B). The five known miRNAs with the highest TPM values were vvi-miR3634-3p, vvi-miR166c, vvi-miR159c, vvi-miR482, and vvi-miR398b, and those of novel miRNAs were 1_21167, 1_21167*, 14_37516, Un_39994*, and 11_7793. 


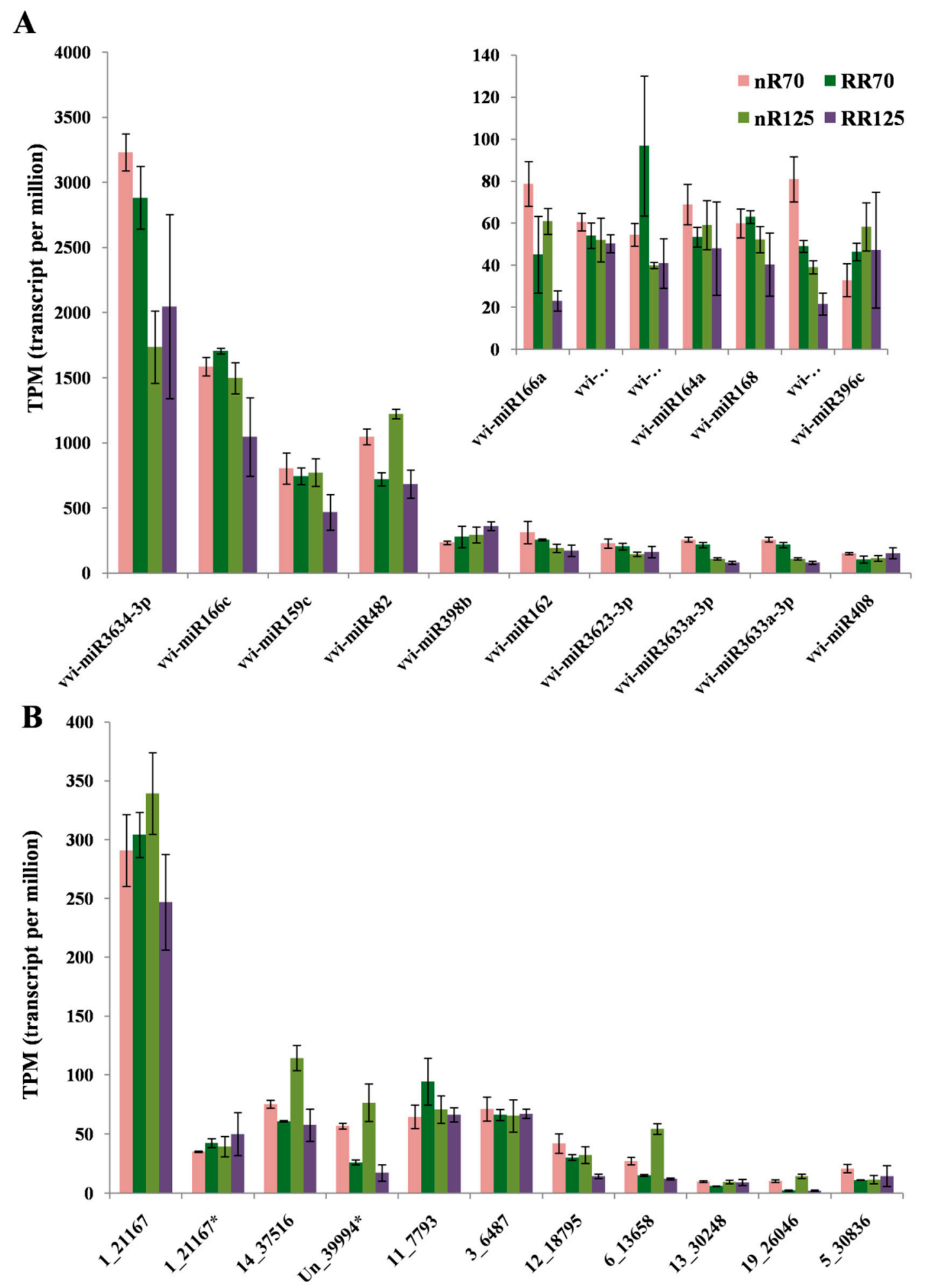

Figure 5. High transcript per million (TPM) values of known miRNAs (A) and novel miRNAs (B) in four root sequencing samples. nR70, RR70, nR125 and RR125 represented the 70 days after planting, simplified DAP and 125 DAP sampling time points under control and root restriction cultivation. 


\subsection{Analysis of voi-miRNA Mediated Grapevine Root Formation}

The number of predicted target genes' mRNAs in DEMs was 344, 738, 402, and 486 in different cultivations (RR70 vs. nR70; RR125 vs. nR125) and different cultivation stages (nR125 vs. nR70; RR125 vs. RR70), respectively. GO annotation analysis revealed that the predicted target genes' mRNAs participated in a variety of biological processes, and both included regulation of transcription, oxidation-reduction processes, serine family amino acid metabolic processes, and defense response. In different cultivation models, the target genes' mRNAs had predicted functions in lignin catabolic processes, electron transport, and response to water deprivation (Figure 6A). In addition, response to abscisic acid stimulus, response to salt stress, regulation of meristem growth, and polarity specification of adaxial/abaxial axes were ranked in the top 10 for different cultivation stages (Figure 6B). The first category in cellular component classification was the nucleus, while the protein binding and ATP binding categories were the most abundant categories in molecular function classification. Gene function annotation found a total of 24 target genes' mRNAs related to root development, which corresponded to 17 vvi-miRNAs. Target genes' mRNAs of vvi-miR156, vvi-miR166, vvi-miR2111-5p, and vvi-miR3624-3p participated in root hair development, and vvi-miR164 and vvi-miR482 affected lateral root and root cap development. Target genes' mRNAs of vvi-miR396 were annotated in root development. Target genes' mRNAs of novel miRNAs had more functions related to root development. For example, the target genes' mRNAs of 4_24249 and 17_2431 affected primary root development, while those of 15_8868 and 15_8867 participated in root morphogenesis. KEGG analysis was conducted and some target gene mRNAs had corresponding metabolic pathway annotations. Among them, miR2111-5p participated in vasopressin-regulated water re-absorption, corresponding to its GO annotation in root hair development (Table 3). 
$\mathbf{A}$
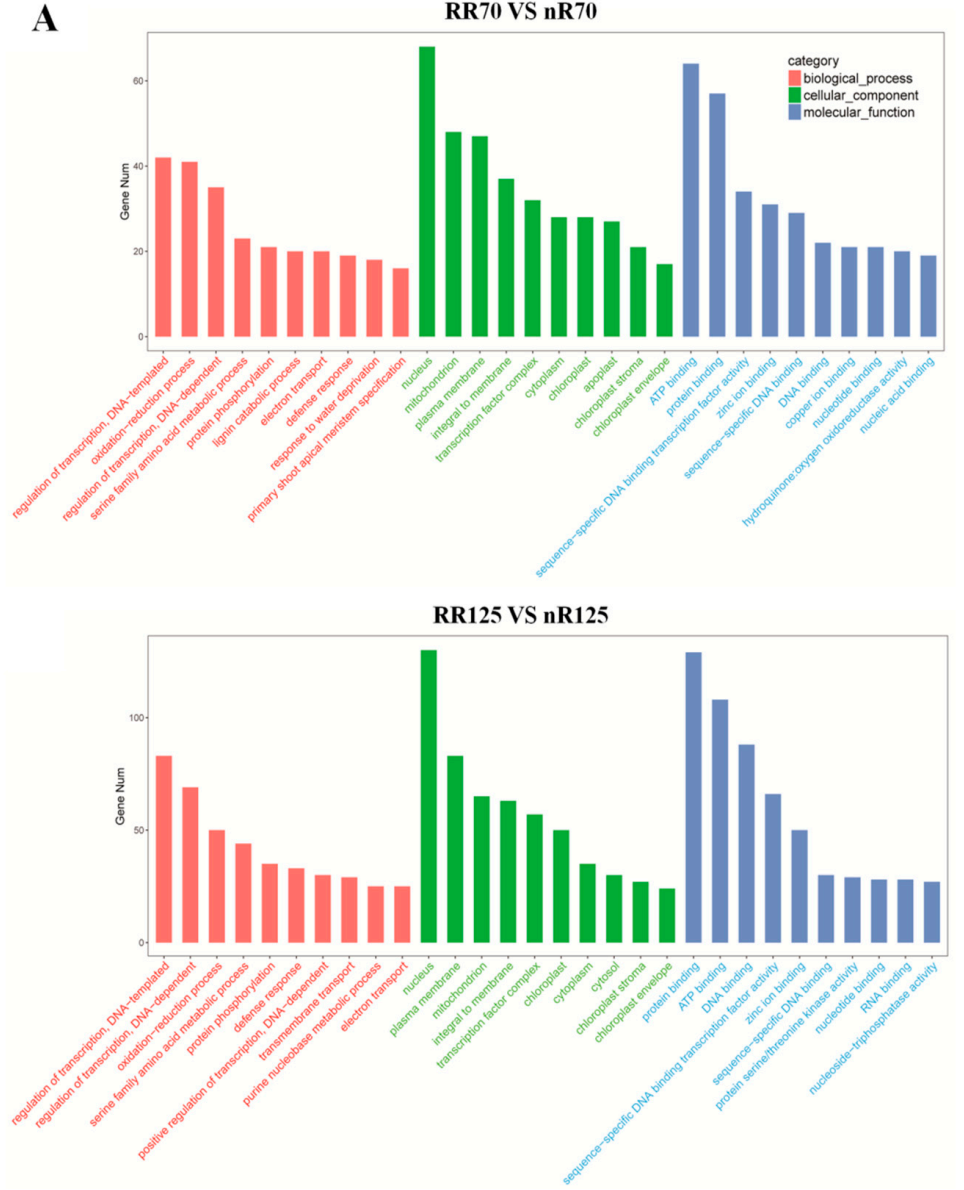

B

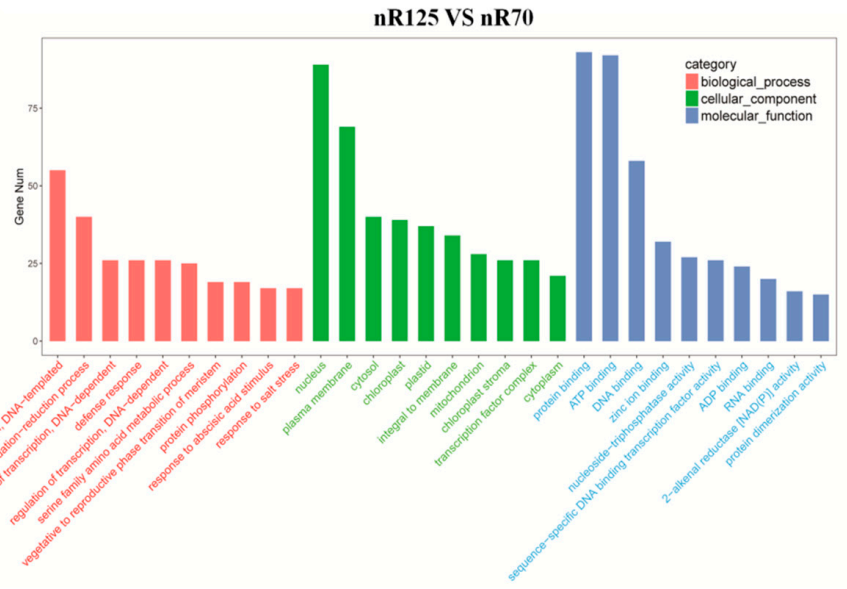

RR125 VS RR70

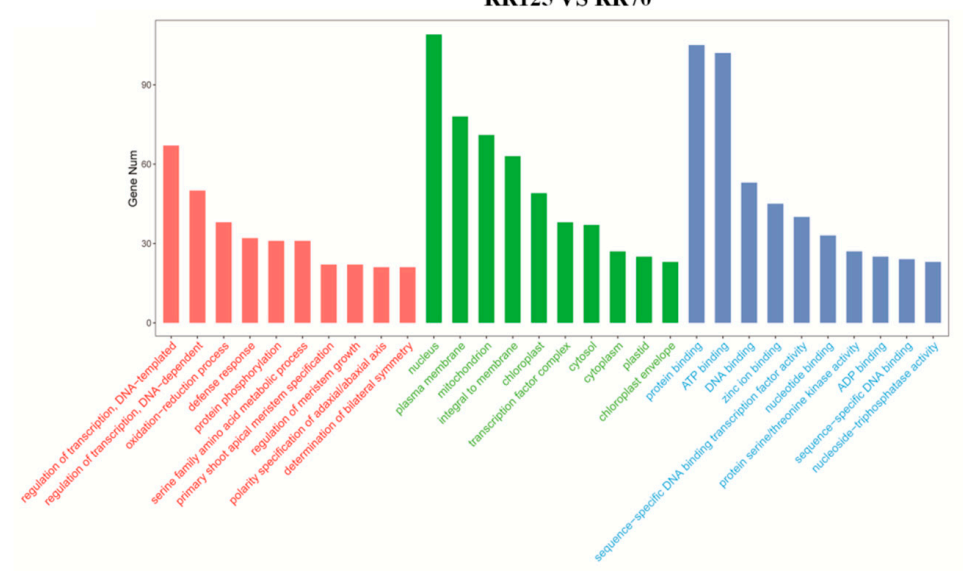

Figure 6. Gene ontology analysis of the predicted target genes of the differentially expressed miRNAs between different cultivations (A) and different cultivation stages (B) in four root sequencing samples. nR70, RR70, nR125 and RR125 represented the 70 days after planting, simplified DAP and 125 DAP sampling time points under control and root restriction cultivation. 
Table 3. Predicted target genes' mRNA of differentially expressed miRNAs related to root development.

\begin{tabular}{|c|c|c|c|c|c|c|c|c|c|c|c|}
\hline MiRNA_id & $\begin{array}{l}\text { Target Gene' } \\
\text { mRNA }\end{array}$ & $\begin{array}{c}\text { Target } \\
\text { Start }\end{array}$ & $\begin{array}{c}\text { Target } \\
\text { End }\end{array}$ & Strand & Score & Target Seq $\left(5^{\prime}->3^{\prime}\right)$ & Query Seq $\left(3^{\prime}->5^{\prime}\right)$ & GO id & Gene Function Annoation & KEGG Pathway & KEGG Pathway Description \\
\hline $4 \_24249$ & VIT_214s0068g01330 & 3178 & 3196 & + & 4 & $\begin{array}{l}\text { AAACGGAAGA } \\
\text { ACCACCAUA }\end{array}$ & $\begin{array}{l}\text { AUAGCCUUAUU } \\
\text { GGUGGUAU }\end{array}$ & GO:0080022 & primary root development & NA & NA \\
\hline 17_2431 & VIT_212s0057g00680 & 1326 & 1346 & + & 4 & $\begin{array}{l}\text { GGAGUUCCUGA } \\
\text { GUCAGCAGCU }\end{array}$ & $\begin{array}{l}\text { CCCUAGGUAU } \\
\text { UCAGUCGUCGA }\end{array}$ & $\begin{array}{l}\text { GO:0080022; } \\
\text { GO:0010071; } \\
\text { GO:0010078 }\end{array}$ & $\begin{array}{c}\text { primary root development } \\
\text { |root meristem specification | } \\
\text { maintenance of root meristem } \\
\text { identity }\end{array}$ & NA & NA \\
\hline $\begin{array}{l}15 \_8868> \\
15 \_8867\end{array}$ & VIT_208s0058g01340 & 2392 & 2409 & + & 3 & $\begin{array}{l}\text { CAUGCUGCU } \\
\text { GCCAGCCCA }\end{array}$ & $\begin{array}{l}\text { CCACCACGA } \\
\text { CGGUCGGGU }\end{array}$ & GO:0016021 & root morphogenesis & NA & NA \\
\hline $\begin{array}{l}15 \_8868> \\
15 \_8867\end{array}$ & VIT_211s0037g00040 & 1169 & 1185 & + & 4 & $\begin{array}{l}\text { GGUGGUGCU- } \\
\text { CCAGCACA }\end{array}$ & $\begin{array}{l}\text { CCACCACGA } \\
\text { CGGUCGGGU }\end{array}$ & GO:0080147 & root hair cell development & NA & NA \\
\hline 6_12672* & VIT_203s0063g02440 & 545 & 562 & + & 4 & $\begin{array}{c}\text { ACC-CCCGU } \\
\text { AUUAUUCAUA }\end{array}$ & $\begin{array}{l}\text { UGGAGGGCGU } \\
\text { AGUAAGUGU }\end{array}$ & GO:0048364 & root development & NA & NA \\
\hline 6_13011 & VIT_211s0016g01900 & 119 & 136 & + & 4 & $\begin{array}{l}\text { AAGUCAUCGC } \\
\text { A-CACAGCA }\end{array}$ & $\begin{array}{l}\text { UUCAGUAGA } \\
\text { GUAGUGUCGU }\end{array}$ & GO:0048528 & $\begin{array}{l}\text { post-embryonic root } \\
\text { development }\end{array}$ & NA & NA \\
\hline 9_20003 & VIT_214s0066g00370 & 1930 & 1947 & + & 4 & $\begin{array}{l}\text { GCUGUCUUG- } \\
\text { CCACCAGAG }\end{array}$ & $\begin{array}{l}\text { UGGCGGAACU } \\
\text { GGUGGUCUU }\end{array}$ & GO:0010053 & $\begin{array}{l}\text { root epidermal cell } \\
\text { differentiation }\end{array}$ & NA & NA \\
\hline 9_20003 & VIT_214s0066g00370 & 1930 & 1947 & + & 4 & $\begin{array}{l}\text { GCUGUCUUG-C } \\
\text { CACCAGAG }\end{array}$ & $\begin{array}{l}\text { UGGCGGAAC } \\
\text { UGGUGGUCUU }\end{array}$ & GO:0010053 & $\begin{array}{l}\text { root epidermal cell } \\
\text { differentiation }\end{array}$ & NA & NA \\
\hline 19_25758 & VIT_201s0010g03300 & 3037 & 3054 & + & 4 & $\begin{array}{l}\text { GAGGCAUCCG } \\
\text { UAUUUUCA }\end{array}$ & $\begin{array}{l}\text { CUCGGUAGG } \\
\text { CGUCAAAGU }\end{array}$ & GO:0010053 & $\begin{array}{l}\text { root epidermal cell } \\
\text { differentiation }\end{array}$ & ko00510, ko04141 & $\begin{array}{l}\text { N-Glycan biosynthesis | } \\
\text { Protein processing in } \\
\text { endoplasmic reticulum }\end{array}$ \\
\hline 19_24713 & VIT_213s0019g02390 & 1712 & 1728 & + & 4 & $\begin{array}{l}\text { UCAUUCA-C } \\
\text { UCAAAAUCU }\end{array}$ & $\begin{array}{l}\text { AGUCAGUUG } \\
\text { GGUUUUAGA }\end{array}$ & $\begin{array}{l}\text { GO:0048767; } \\
\text { GO:0010449 }\end{array}$ & $\begin{array}{l}\text { root hair elongation | root } \\
\text { meristem growth }\end{array}$ & ko00790 & Folate biosynthesis \\
\hline 19_24713 & VIT_213s0019g04320 & 129 & 146 & + & 4 & $\begin{array}{l}\text { UUGUUCAAC } \\
\text { CCCAAAUCU }\end{array}$ & $\begin{array}{l}\text { AGUCAGUUG } \\
\text { GGUUUUAGA }\end{array}$ & GO:0048765 & root hair cell differentiation & NA & NA \\
\hline 19_24713 & VIT_203s0038g03620 & 4817 & 4833 & + & 4 & $\begin{array}{l}\text { UCAG-CAACC } \\
\text { AAAAAUCA }\end{array}$ & $\begin{array}{l}\text { AGUCAGUUG } \\
\text { GGUUUUAGA }\end{array}$ & GO:0048767 & root hair elongation & NA & NA \\
\hline vvi-miR3624-3p & VIT_205s0029g00330 & 1110 & 1130 & + & 4 & $\begin{array}{l}\text { AGGGCUGCGCU } \\
\text { GCUGCCCUGA }\end{array}$ & $\begin{array}{l}\text { UCAUCAUACG } \\
\text { ACGACGGGACU }\end{array}$ & GO:0005829 & root hair elongation & ko00270 & $\begin{array}{l}\text { Cysteine and methionine } \\
\text { metabolism }\end{array}$ \\
\hline 2_4979* & VIT_218s0001g04980 & 889 & 907 & + & 4 & $\begin{array}{l}\text { GGAUGAAUGA } \\
\text { GUCGG-AGAU }\end{array}$ & $\begin{array}{l}\text { CCUACUUACUC } \\
\text { AGCCGUCGA }\end{array}$ & GO:0048364 & root development & $\begin{array}{c}\text { ko01212, } \\
\text { ko00620, } \\
\text { ko00640, } \\
\text { ko00061, } \\
\text { ko00254, ko04152 }\end{array}$ & $\begin{array}{c}\text { Fatty acid metabolism | } \\
\text { Pyruvate metabolism | } \\
\text { Propanoate metabolism | } \\
\text { Fatty acid biosynthesis | } \\
\text { Aflatoxin biosynthesis | } \\
\text { AMPK signaling pathway }\end{array}$ \\
\hline 2_4979* & VIT_207s0289g00100 & 395 & 413 & + & 4 & $\begin{array}{l}\text { GGAUGAAUGAG } \\
\text { UCGG-AGAU }\end{array}$ & $\begin{array}{l}\text { CCUACUUACU } \\
\text { CAGCCGUCGA }\end{array}$ & GO:0048364 & root development & NA & NA \\
\hline
\end{tabular}


Table 3. Cont

\begin{tabular}{|c|c|c|c|c|c|c|c|c|c|c|c|}
\hline vvi-miR396 & VIT_208s0007g03760 & 703 & 723 & + & 3 & $\begin{array}{c}\text { CGUUCAAGAA } \\
\text { AGCCUGUGGAA }\end{array}$ & $\begin{array}{l}\text { UCAAGUUCUU } \\
\text { UCG-ACACCUU }\end{array}$ & GO:0048364 & root development & NA & NA \\
\hline vvi-miR2111-5p & VIT_208s0007g01270 & 1635 & 1655 & + & 0 & $\begin{array}{l}\text { UAGACCUCAGG } \\
\text { AUGCAGAUUA }\end{array}$ & $\begin{array}{l}\text { AUCUGGAGUC } \\
\text { CUACGUCUAAU }\end{array}$ & GO:0080147 & root hair cell development & ko04962 & $\begin{array}{l}\text { Vasopressin-regulated water } \\
\text { re-absorption }\end{array}$ \\
\hline vvi-miR156 & VIT_204s0008g00960 & 2493 & 2512 & + & 1 & $\begin{array}{l}\text { GUGCUCACUC } \\
\text { UCUUCUGUCA }\end{array}$ & $\begin{array}{l}\text { CACGAGUGAG } \\
\text { AGGAGACAGU }\end{array}$ & GO:0048767 & root hair elongation & ko04010, ko04020 & $\begin{array}{l}\text { MAPK signaling pathway | } \\
\text { Calcium signaling pathway }\end{array}$ \\
\hline vvi-miR166 & VIT_209s0002g03740 & 1510 & 1528 & + & 0.5 & $\begin{array}{l}\text { GGAAUGAAG } \\
\text { CCUGGUCCGG }\end{array}$ & $\begin{array}{l}\text { CCUUACUUCG } \\
\text { GACCAGGCU }\end{array}$ & GO:0048765 & root hair cell differentiation & NA & NA \\
\hline vvi-miR166 & VIT_206s0004g02800 & 1730 & 1748 & + & 1 & $\begin{array}{l}\text { GGGAUGAAG } \\
\text { CCUGGUCCGG }\end{array}$ & $\begin{array}{l}\text { CCUUACUUCG } \\
\text { GACCAGGCU }\end{array}$ & GO:0045595 & root hair cell differentiation & NA & NA \\
\hline vvi-miR166 & VIT_213s0019g04320 & 1114 & 1132 & + & 1 & $\begin{array}{l}\text { GGGAUGAAG } \\
\text { CCUGGUCCGG }\end{array}$ & $\begin{array}{l}\text { CCUUACUUCG } \\
\text { GACCAGGCU }\end{array}$ & GO:0008289 & root hair cell differentiation & NA & NA \\
\hline vvi-miR166 & VIT_209s0002g03740 & 1508 & 1528 & + & 2.5 & $\begin{array}{l}\text { CUGGAAUGAAG } \\
\text { CCUGGUCCGG }\end{array}$ & $\begin{array}{l}\text { CCCCUUACUU } \\
\text { CGGACCAGGCU }\end{array}$ & GO:0048263 & root hair cell differentiation & NA & NA \\
\hline vvi-miR164 & VIT_219s0027g00230 & 589 & 609 & + & 1.5 & $\begin{array}{c}\text { AGCAAGUGCC } \\
\text { CUGCUUCUCCG }\end{array}$ & $\begin{array}{l}\text { UCGUACACGG } \\
\text { GACGAAGAGGU }\end{array}$ & GO:0048527 & lateral root development & NA & NA \\
\hline vvi-miR482 & VIT_218s0001g03540 & 372 & 392 & + & 4 & $\begin{array}{l}\text { GGAGUGAGAG } \\
\text { GAG-AGGAAAGG }\end{array}$ & $\begin{array}{l}\text { CCUUACCCUCCU } \\
\text { CAUCCUUUCU }\end{array}$ & $\begin{array}{l}\text { GO:0010311; } \\
\text { GO:0048829 }\end{array}$ & $\begin{array}{l}\text { lateral root formation | root } \\
\text { cap development }\end{array}$ & ko04075 & $\begin{array}{l}\text { Plant hormone signal } \\
\text { transduction }\end{array}$ \\
\hline
\end{tabular}




\subsection{Vvi-miR160 Family Contributes to Grapevine Root Development}

Root tip degradation was one of the most obvious root phenotypes after RRC. MiR160 had been reported to play an important role in root tip development [28]. Five members of the vvi-miR160 family, named vvi-miR160a, b, c, d, and e were obtained using a miRBase search. Among them, the mature sequence lengths of vvi-miR160a and vvi-miR160b were $23 \mathrm{bp}$, and those of vvi-miR160c, $d$, and e were $21 \mathrm{bp}$. There was one base difference between the overlap of vvi-miR160 mature sequences. MiR160 precursor sequence alignment result showed that the flanking sequence was variable, but that the mature sequence was similar. Moreover, the mature sequences of vvi-miR160c, $d$, and e were 21 bp, which was the same with ath-miR160 (Figure 7A). Phylogenetic analysis of miR160 precursors revealed that vvi-miR160a and vvi-miR160b were clustered into one branch, while vvi-miR160c, d, and e were clustered into another branch, and vvi-miR160c was close to ath-miR160c (Figure 7B). Additionally, RNAfold software was used to predict the stem-loop secondary structures of vvi-miR160 family members according to their precursors, and the vvi-miR160c obtained the highest free energy (Figure 7C). Small RNA sequencing detected vvi-miR160 at a moderate expression level in different sequencing samples, but there was no differential expression among samples (Figure 7D). Quantitative analysis of the relative expression of vvi-miR160 precursors found that vvi-miR160c had the highest expression number, followed by vvi-miR160b, and the expression of vvi-miR160a precursor was not detected (Figure 7E).

A

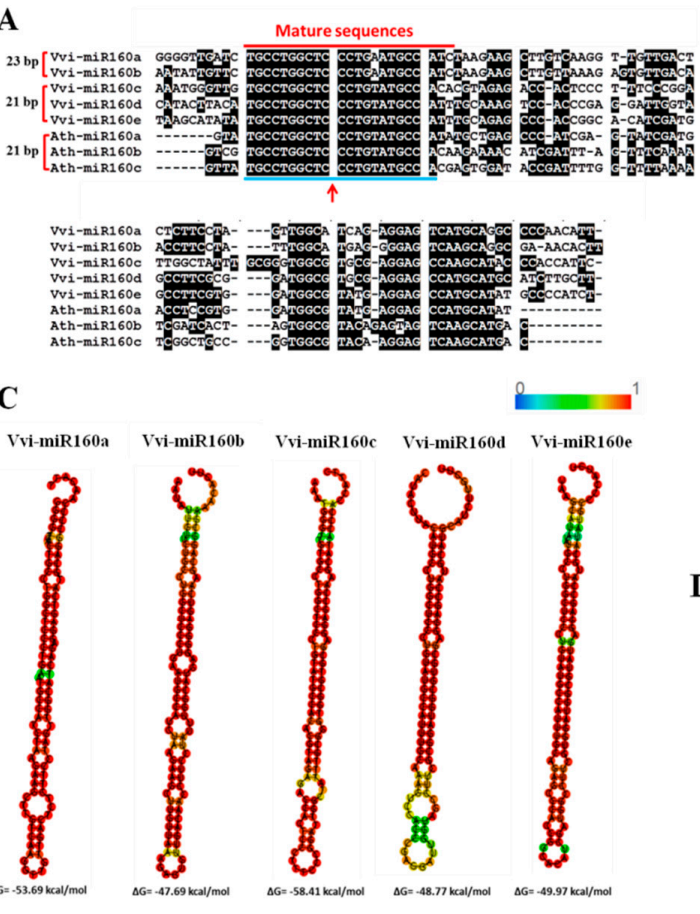

B

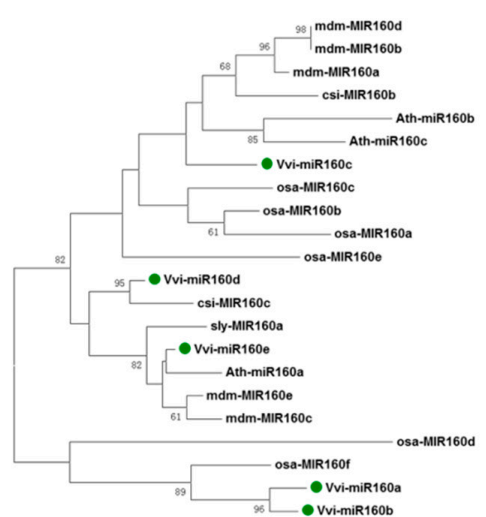

D

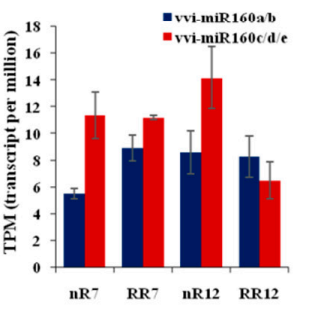

$\mathbf{E}$

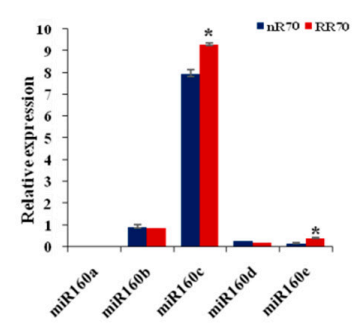

Figure 7. Structural analysis of the vvi-miR160 family. (A) Sequence alignment analysis of miRNA precursor between vvi-miR160 and ath-miR160. (B) Phylogenetic analysis of miR160 in different species. (C) The secondary structure of different vvi-miR160 members. (D)Transcript per million (TPM) values ofvvi-miR160 members in four root sequencing samples. (E) The relative expression of the precursor of vvi-miR160 members in control and root restriction cultivation at 70 DAP sampling time point; nR70, RR70, nR125 and RR125 represented the 70 days after planting, simplified DAP and 125 DAP sampling time points under control and root restriction cultivation, respectively. Error bars indicate the mean \pm SD $(n=3)$ with biological replicates, and values $\left(^{*}\right)$ are statistically significant from the control cultivation based on Student's $t$ test $(P<0.05)$. 


\section{Discussion}

Assessing the quality of the small RNA sequencing, there were 186 grapevine miRNAs recorded in the miRBase database, and in this work, 153 of them were detected in grapevine root, with a high detection rate of $82.3 \%$, indicating the crucial role of miRNAs in grapevine root initialization and development (Figure 3). Principal component analysis revealed a close distance between nR70 and RR70 whereas nR125 and RR125 were completely separated (Supplementary Materials Figure S6), which was consistent with the more notable root phenotypic difference in the 12th sampling. Vvi-miR828 had been reported to play a role in the anthocyanin metabolism pathway and to affect fruit coloring [29]. The absence of vvi-miR828 in grapevine root was reasonable, as no anthocyanin accumulation in the root. MiRNA length was considered to be 19-24 nts and usually showed a typical $21 \mathrm{nts}$ and $24 \mathrm{nts}$ two-peak distribution in small RNA sequencing results. In previous research, it was reported that the 24 nts length small RNA was most abundant in grapevine flowers and flower organs (carpels and stamens), and there was no significant peak in miRNA length distribution in seeds [25]. However, the remaining tissues, including roots, had the highest proportion of $21 \mathrm{nts}$ sequences. In this study, the $21 \mathrm{nts}$ sequence had the largest content in known miRNAs (Figure 2A), while novel miRNAs had two peaks at $21 \mathrm{nts}$ and $23 \mathrm{nts}$ in length, while the proportion of $24 \mathrm{nts}$ was relatively small (Figure 2B), which was different from previous studies.

Understanding the root phenotypes of plants under RRC requires further study to determine the molecular mechanisms of root development. Comparing the root system after root restriction cultivation with previous studies, we summarized the characteristics of root development under different cultivation models through continuous observation in grapevine (Vitis vinifera L. cv. Muscat Hamburg). Finally, the differences between the two root systems could be attributed to two basic phenotypes, namely the degradation of the root tip and the occurrence of a large number of adventitious roots and lateral roots (Figure 1). The root tip includes the root cap, root meristem, and the root distal region, which showed complex behavioral patterns such as decision-making and played an important role in plant gravitropism. Ath-miR160 has been reported to be involved in root elongation and root cap formation [28]. Overexpression of ath-miR160c displayed uncontrolled cell division in the root distal region and loss of gravity-sensing. Moreover, the root length of the seedling was reduced, the lateral root number was increased, and its target gene's arf10arf16 double mutant showed the same phenotype. Phylogenetic analysis revealed that vvi-miR160c was in the same clade as ath-miR160c (Figure 7), which indicated vvi-miR160c may influence root tip development in grapevine. In addition, multiple miRNAs have been reported to participate in lateral root development in plants (Figure 8). For example, miR156 targeted Spls [30], miR164 targeted NAC [18], miR396 targeted bHLH74 [31], and miR171 targeted GRAS [32] to regulate lateral root development. These miRNAs involved in lateral root development were differentially expressed in at least one of the root development stages in grapevine. Among them, miR167 was reported to negatively regulate the numbers of lateral roots. In this study, miR167a was up-regulated, while miR167b was down-regulated after RRC, indicating that functional differences exist among miRNA family members. The down-regulated expression of miR $167 \mathrm{~b}$ is in line with the characteristics of promoting lateral root development, which deserves further study. Adventitious roots are an indispensable feature of large-scale plant reproduction. MiRNAs affected the development of adventitious roots by targeting auxin response factors [33]. In Arabidopsis, ath-miR160 can target auxin response factor AtARF17 to regulate the occurrence of adventitious roots, in which the number of adventitious roots increased after overexpression of ath-miR160 in Arabidopsis, while the number of adventitious roots decreased in plants overexpressing AtARF17.AtARF6 and AtARF8 are positive regulators that promote adventitious root growth, and both are strictly regulated by ath-miR167. Moreover, it was found that AtARF6, AtARF8, and AtARF17 were functionally redundant and had an additive effect in regulating the occurrence of adventitious roots. Overexpression of AtARF17 in the background of arf6arf8 mutants had the least number of adventitious roots. Combined with ath-miR160 targeted AtARF10 and AtARF16 that affected root tip development, miR160 was indispensable for the overall development of the root system. Although vvi-miR160c showed no 
difference in expression of root development after RRC, the role of vvi-miR160 is still worthy of further study.

The root system explores the soil for nutrients. In this exploration process, due to space limitations, the relative soil amount of the root system decreased. Therefore, in the case of insufficient soil, the root system may supplement the deficiency of root tip degradation by issuing more lateral roots, which increases the root surface area and helps in the search for more soil and nutrients for growth. With continuous growth of the root system, the roots become thinner. Root restriction cultivation is the spatial stress response to growth of the root system. In this study, some differentially expressed miRNAs after RRC were rarely reported to participate in root development, but played a role in biotic or abiotic stresses (Figure 8). Among them, miR482 was a miRNA related to disease resistance [34,35], which is a highly conserved miRNA and only found in some species. MiR2111 was detected in the phloem sap under phosphorus-limited conditions, and the abundance of miR2111 in the phloem sap of Brassica napus strongly depended on the concentration of $\mathrm{P}$ or $\mathrm{N}$, suggesting that it was effective at low phosphorus in plant roots [36]. In addition, miR167 targeted IAR3 [37], miR169 targeted NFYAs [38], miR398 targeted CSDS [39], and miR408 [40] was involved in drought stress. All these results indicate that RRC is part of a combination of multiple stress processes, and the effect of drought was obvious. Meanwhile, several newly discovered miRNA family members, namely miR3623-3p, miR3627-3p, miR3632-3p, miR3633a-3p, and miR3634-3p, were also detected and were also highly and differentially expressed, but their functions are still unclear and needed further study.

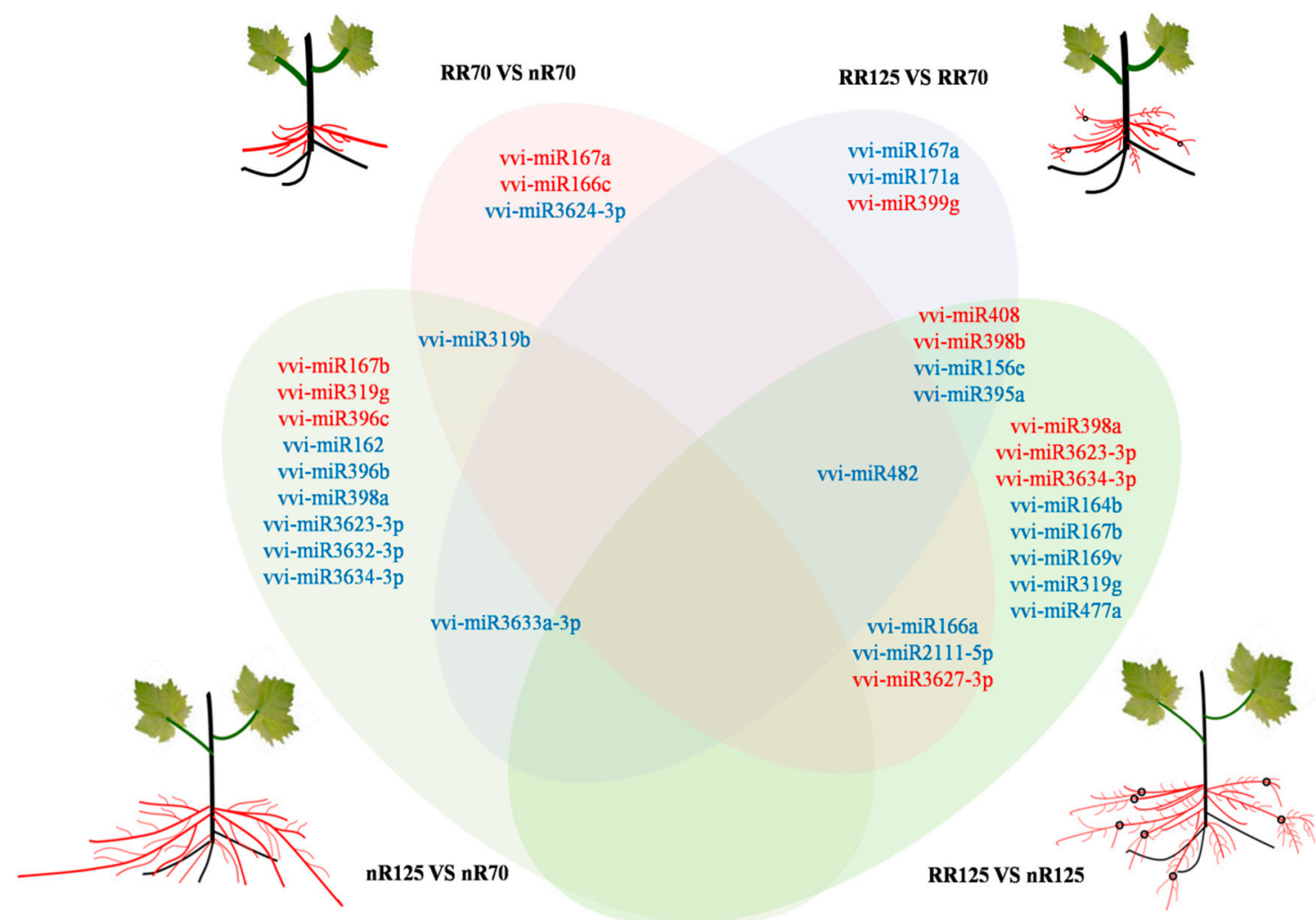

Figure 8. Overview of the root architecture model and miRNAs regulating root formation in grapevine under root restriction cultivation (RRC). The red lines indicate the newly emerging roots, and the black circles indicate the degenerated positions of the root tips after root restriction cultivation; different color ovals represent different sample comparison groups: pink represents RR70 vs. nR70, blue represent RR125 vs. RR70, yellow represents nR125 vs. nR70, green represents RR125 vs. nR125, and the overlay is the intersection of different comparison groups. nR70, RR70, nR125 and RR125 represent the 70 days after planting, simplified DAP and 125 DAP sampling time points under control (nRC) and RRC. 


\section{Materials and Methods}

\subsection{Plant Materials}

The grapevine materials used in this study were cuttings. One-year-old Vitis vinifera L. cv. Muscat Hamburg with roots that formed in the autumn of 2016 were planted in the greenhouse of the Fruit Tree Laboratory in Shanghai Jiao Tong University $\left(31^{\circ} 11^{\prime} \mathrm{N}, 121^{\circ} 29^{\prime} \mathrm{W}\right)$ in 2017 . Two different cultivation models including Root restriction cultivation (referred to RRC) and control cultivation (referred to $\mathrm{nRC}$ ) were used in this study. In root restriction cultivation, 100 cuttings were cultivated in the root zone container with a diameter of $30 \mathrm{~cm}$ and a height of $30 \mathrm{~cm}$ (with holes around it) and separated from the ground by a tray. The planting substrate was soil, organic fertilizer and perlite with 1:1:1 mixed. In control cultivation, 100 plants were planted on the ground with a height of $40 \mathrm{~cm}$ in the same substrate. The initial planting distance was $70 \mathrm{~cm} \times 70 \mathrm{~cm}$. Sampling was performed in a zigzag pattern to ensure that there was sufficient space for root development. The above-ground management were the same, and all of them maintain single-vine growth with no topping. The secondary shoots were trimmed every 7_10 days. Moreover, the experimental materials were equipped with unified control irrigation measures. The roots firstly sampled on April 24, 2017 when above-ground started to sprout, and then roots were sampled at 10-day intervals during the first 11 time points and at 15-day intervals at the last time point. Finally, 24 grapevine root samples from two different cultivation models at 12 time points $(10,20,30,40,50,60,70,80,90,100,110$, and 125 days after planting) were collected. At each sampling time point, 6_9 trees were selected as biological replicates.

\subsection{Small RNA Libraries Construction and Illumina Sequencing}

According to root morphology observation, small RNA sequencing was performed on four root samples from the 70 DAP and 125 DAP sampling time points under two cultivation conditions. Total RNA was extracted from collected root samples using CTAB method. RNA concentration and quality were detected using both NanoDrop 2000 (Thermo Scientific, Waltham, MA, USA) and Agilent 2100 Bioanalyzer (Agilent Technologies, Palo Alto, CA, USA). 12 small RNA Libraries (four samples each with three biological replicates) were constructed according to the TruSeq Small RNA Sample Preparation Guide kit (Illumina, Foster City, CA, USA) and sequenced using Illumina Hiseq 2000. Briefly, $1 \mu \mathrm{g}$ of total RNA was ligated to the 3'- and 5'-sequencing adapters by T4 RNA ligase and reversed into cDNA by Super Script III reverse transcriptase (Invitrogen, New York, NY, USA). The obtained cDNA template was PCR amplified using adaptor primers for 15 cycles. The product was separated and purified by $6 \%$ Novex TBE polyacrylamide gel electrophoresis (Invitrogen, New York, NY, USA), and RNA fragments in the range of 147-157 nts were excised and recovered. The length and quality of the library were determined by Agilent 2100 bioanalyzer. The RNA-Sequencing raw data have been deposited to the National Centre for Biotechnology Information (NCBI) BioProject database under accession number PRJNA601829. All the supporting data are included as additional files.

\subsection{Identification of Known and Novel voi-miRNAs}

The criteria for the raw data quality control included: (1) remove the adapter sequence by cutadapt [41] software, and filter sequences less than $15 \mathrm{bp}$ and greater than $41 \mathrm{bp}$ in length; (2) use fastx_toolkit [42] software to perform Q20 quality control and retain sequences with Q20 above 80\%; (3) filter reads containing N bases by NGSQCToolkit [43] and get clean reads; (4) remove redundant sequence and obtained clean reads uniq.

The process to obtain known and novel vvi-miRNAs contained the following steps: (1) Clean reads were mapped to the reference $V$. vinifera L. cv. Pinot Noir (PN40024) genomes to remove unmapped reads. (2) compared the filtered reads with Rfam [44] (version 10.0) database by blastn [45], extracted the results with E-value $\leq 0.01$, annotates and removed the sequences such as rRNA, snRNA, snoRNA, tRNA; (3) remove sequences perfect matched with the transcript and longer than 26 bp and less than 15 bp by bowtie [46] software; (4) remove redundant sequence by RepeatMasker [47] software. 
After the filtration and removal steps, the remaining sequences were perfect matched with mature miRNAs database in the miRBase [48], Sequences with perfect matches were considered as known miRNAs in V.vinifera. The unannotated sRNAs were performed secondary structure prediction by RNAfold [49] database, and sequences with miRNA hairpins were considered as novel miRNAs.

Moreover, the predicted novel miRNAs were searched in the miRVIT [27] database to annotate novel miRNAs that had been reported in grapevine before. In addition, the novel miRNA also aligned with mature miRNAs database in the miRBase 21 by the default parameter (Evalue cutoff $\leq 10$, Mismatch penalty $=-4$, Match score $\geq 60$ ). Aligned novel miRNAs were considered as non-conserved miRNAs, while unaligned ones were considered as grapevine specific novel miRNAs.

\subsection{Differentially Expressed miRNAs Analysis and Annotation of the Target Genes}

MiRNA expression quantification was normalized according to the expression of transcript per million (TPM) [50] and calculated as: TPM = Reads count of per miRNA/Reads count of per miRNA $\times 10^{6}$. DESeq [51] (v1.18.0) software in the R package was used for miRNA differential expression analysis, the $p$ value was calculated, and miRNAs with a $P$ value $<0.05$ were selected. At the same time, hierarchical clustering analysis using the Euclidean distance measurement with $\mathrm{MeV}$ software was performed on differentially expressed miRNAs between different samples. Target genes were predicted using targetfinder [52] software, and GO functional annotation and KEGG analysis were performed.

\subsection{Structure Analysis of voi-miR160 Family}

The miR160 family members and sequence information of grapevine and Arabidopsis were retrieved by NCBI (www.ncbi.nlm.nih.gov), and the obtained sequences were aligned and analyzed by BioEdit [53] software. In addition, vvi-miR160 was blast in NCBI to find miR160 sequences with high homology to other species. MEGA 6 [54] software was used for phylogenetic analysis of miR160 in different species. The RNAfold [49] was used to predict the secondary structure of different vvi-miR160 members. Gene accession number listed in Supplementary Materials Table S5

\subsection{RT-qPCR Analysis of the Expression Levels of voi-miR160 Family}

RNA extraction of grapevine root was performed according to the instructions of TianGen RNAprep Pure Plant Kit (TIANGEN, Beijing, China, DP441). RNA quality was detected by $1.2 \%$ agarose gel electrophoresis and a NanoDrop 2000 system (Thermo Scientific, Waltham, MA, USA) to ensure RNA OD 260/280 values ranged from 1.8 to 2.0. First-strand cDNA was generated according to the steps of PrimeScript ${ }^{\mathrm{TM}}$ RT Master Mix (TaKaRa, Dalian, China, RR036A). Primer Premier 5 software was used for gene-specific primer design (Supplementary Materials Table S5). The RT-qPCR was performed on a CFX Connect Real-Time Detection System (Bio Rad, Hercules, USA). A 10- $\mu$ L reaction volume included $5 \mu \mathrm{L}$ of TB Green Premix Ex Taq II (TAKARA, Dalian, China), $0.5 \mu \mathrm{L}$ of each primer $(10 \mu \mathrm{M}), 1 \mu \mathrm{L}$ of cDNA template (diluted 10-fold), and $3 \mu \mathrm{L}$ of double-distilled water. Two-step RT-qPCR was performed using the following conditions: initial denaturation at $95^{\circ} \mathrm{C}$ for $150 \mathrm{~s}$, followed by 40 cycles at $95^{\circ} \mathrm{C}$ for $5 \mathrm{~s}$ and $60^{\circ} \mathrm{C}$ for $30 \mathrm{~s}$. The $V v G A P D H$ (XM_002263109.3) was used as an internal control [55]. The relative expression of the genes was calculated using the $2^{-\Delta \Delta C t}$ method. Each experiment was repeated three times.

\subsection{Statistical Analyses}

Statistical significance was evaluated by Student's $t$ test analysis.

\section{Conclusions}

Grapevine root architecture had been changed in root restriction cultivation after planting for 70 days, which was mainly manifested as root tip degradation, subsequently caused a large number of lateral roots, and also enhanced the rate of root regeneration. Small RNA sequencing was performed 
on the 70 DAP and 125 DAP sampling time points of the root restriction cultivation and control. A total of 153 known miRNAs and 119 predicted novel miRNAs were obtained. Annotations of the novel miRNAs by miRVIT and miRbase database obtained 14 known new miRNA members and 23 grapevine-specific miRNAs. Differentially expressed miRNAs analysis found that multiple miRNAs were reported to be involved in root system development, and biotic and abiotic stresses, indicating that root restriction cultivation was jointly regulated by multiple miRNAs, and multiple minor stresses exist in root development on root restriction condition. In addition, the specific expression of vvi-miR160c in the apex may be the main cause of apical degradation, which leads to the phenotype of root restriction cultivation.

Supplementary Materials: Supplementary materials can be found at http://www.mdpi.com/1422-0067/21/10/3513/ s1. Figure S1. Aboveground phenotype variations of one-year old grapevine cv. Muscat Hamburg after root restriction cultivation. The length (A) and diameter (B) of shoots in control and root restriction cultivations grew from 60 to 125 days after planting. The grading of secondary shoots in root restriction cultivation (C) and control (D) according to their length. (E) The number of secondary shoots per tree between two pruning. (F) and $(G)$ represent the length and diameter of secondary shoots in different length grades. Error bars indicate the mean \pm SD $(n \geq 10)$ with biological replicates, and values $\left.{ }^{*}\right)$ are statistically significant from the control cultivation based on Student's t test $(P<0.05)$. Figure S2. The root morphology of one-year old grapevine cv. Muscat Hamburg in control cultivation at 12 sampling time points $(10,20,30,40,50,60,70,80,90,100,110$, and 125 days after planting), recorded as nR + DAP. Figure S3. The root morphology of one-year old grapevine cv. Muscat Hamburg in Root restriction cultivation at 12 sampling time points $(10,20,30,40,50,60,70,80,90,100,110$, and 125 days after planting), recorded as nR + DAP. Figure S4. The root updated morphology after root restriction cultivation. White arrows indicate the position of root begun to brown, decay and disappear. Bar $=5 \mathrm{~cm}$. Figure S5. The copy number of read counts in the high throughput sequencing. Figure S6. The principal component analysis of different sequence samples in grapevine. nR70, RR70, nR125 and RR125 represented the 70 days after planting, simplified DAP and 125 DAP sampling time points under control and root restriction cultivation, and each sample had three replicates, recorded as A, B, and C, respectively. Table S1 The information of the predicted novel miRNAs aligned with miRVIT database. Table S2 The information of predicted unconserved grapevine miRNA after alignment with mature sequence in miRBase with default parameters. Table S3 The information of predicted grapevine specific miRNA. Table S4 The information of differentially expressed miRNAs between different cultivation and different cultivation stages. Table S5 The primers and accession number of genes used in this study.

Author Contributions: Conceptualization; H.L., Z.G., L.W., S.S., C.M. and S.W.; methodology; H.L., Z.G.; Formal analysis; H.L., D.L., M.S.Z., and H.U.J.; validation; L.Z., C.Z., S.S., W.X., C.M. and S.W.; writing-original draft preparation; H.L., M.S.Z., and C.M.; writing—review and editing: W.X., C.Z., C.M. and S.W. All authors have read and agreed to the published version of the manuscript.

Funding: This work was supported by National Natural Science Foundation of China $(31701888,31972383)$, the Yunnan Natural Science Foundation (Grant No. 2018FB072), and the Special Funds of Modern Industrial Technology System for Agriculture (CARS-29-zp-7).

Conflicts of Interest: The authors declare no conflict of interest.

\section{Abbreviations}

$\begin{array}{ll}\text { ARF } & \text { Auxin response factor } \\ \text { bHLH } & \text { basic helix-loop-helix } \\ \text { DAP } & \text { Days after planting } \\ \text { DEMs } & \text { Differentially expressed miRNAs } \\ \text { miRNA } & \text { microRNA } \\ \text { NCBI } & \text { the National Centre for Biotechnology Information } \\ \text { ncRNA } & \text { Non-coding RNA } \\ \text { nRC } & \text { Non-Root restriction cultivation } \\ \text { PCA } & \text { Principal component analysis } \\ \text { RRC } & \text { Root restriction cultivation } \\ \text { Spl } & \text { Squamosa-promoter binding protein-like } \\ \text { TAS } & \text { Trans-acting siRNA genes } \\ \text { TPM } & \text { Transcript per million }\end{array}$




\section{References}

1. Tardieu, F. Drought perception by plants do cells of droughted plants experience water stress? Plant Growth Regul. 1996, 20, 93-104. [CrossRef]

2. Hopmans, J.W.; Bristow, K.L. Current capabilities and future needs of root water and nutrient uptake modeling. In Advances in Agronomy; Elsevier: Amsterdam, The Netherlands, 2002; Volume 77, pp. 103-183.

3. Atkinson, J.A.; Rasmussen, A.; Traini, R.; Voss, U.; Sturrock, C.; Mooney, S.J.; Wells, D.M.; Bennett, M.J. Branching Out in Roots: Uncovering Form, Function, and Regulation. Plant Physiol. 2014, 166, 538-550. [CrossRef] [PubMed]

4. Ingram, P.A.; Malamy, J.E. Root system architecture. In Advances in Botanical Research; Elsevier: Amsterdam, The Netherlands, 2010; Volume 55, pp. 75-117.

5. Riaz, S.; Garrison, K.E.; Dangl, G.S.; Boursiquot, J.-M.; Meredith, C.P. Genetic divergence and chimerism within ancient asexually propagated winegrape cultivars. J. Am. Soc. Hortic. Sci. 2002, 127, 508-514. [CrossRef]

6. Li, H.; Gao, Z.; Chen, Q.; Li, Q.; Luo, M.; Wang, J.; Hu, L.; Zahid, M.S.; Wang, L.; Zhao, L.; et al. Grapevine ABA receptor VvPYL1 regulates root hair development in Transgenic Arabidopsis. Plant Physiol. Biochem. PPB 2020, 149, 190-200. [CrossRef] [PubMed]

7. Wang, S.; Okamoto, G.; Hirano, K.; Lu, J.; Zhang, C. Effects of restricted rooting volume on vine growth and berry development of Kyoho grapevines. Am. J. Enol. Vitic. 2001, 52, 248-253.

8. Naor, A.; Gal, Y.; Bravdo, B. Shoot and cluster thinning influence vegetative growth, fruit yield, and wine quality of Sauvignon blanc' grapevines. J. Am. Soc. Hortic. Sci. 2002, 127, 628-634. [CrossRef]

9. Leng, F.; Lin, Q.; Wu, D.; Wang, S.; Wang, D.; Sun, C. Comparative transcriptomic analysis of grape berry in response to root restriction during developmental stages. Molecules 2016, 21, 1431. [CrossRef]

10. Wang, S.; Okamoto, G.; Hirano, K. Effects of rooting-zone restriction on the changes in carbohydrates and nitrogenous compounds in 'Kyoho' grapevines during winter dormancy and early shoot growth. J. Jpn. Soc. Hortic. Sci. 1998, 67, 577-582. [CrossRef]

11. Yu, X.-M.; Li, J.-F.; Zhu, L.-N.; Wang, B.; Wang, L.; Bai, Y.; ZHANG, C.-X.; Xu, W.-P.; Wang, S.-P. Effects of root restriction on nitrogen and gene expression levels in nitrogen metabolism in Jumeigui grapevines (Vitis vinifera L.× Vitis labrusca L.). J. Integr. Agric. 2015, 14, 67-79. [CrossRef]

12. Taft, R.J.; Pang, K.C.; Mercer, T.R.; Dinger, M.; Mattick, J.S. Non-coding RNAs: Regulators of disease. J. Pathol. A J. Pathol. Soc. Great Br. Irel. 2010, 220, 126-139. [CrossRef]

13. Mattick, J.S.; Makunin, I.V. Non-coding RNA. Hum. Mol. Genet. 2006, 15 (Suppl. 1), R17-R29. [CrossRef] [PubMed]

14. Ha, M.; Kim, V.N. Regulation of microRNA biogenesis. Nat. Rev. Mol. Cell Biol. 2014, 15, 509. [CrossRef] [PubMed]

15. Li, S.; Liu, L.; Zhuang, X.; Yu, Y.; Liu, X.; Cui, X.; Ji, L.; Pan, Z.; Cao, X.; Mo, B. MicroRNAs inhibit the translation of target mRNAs on the endoplasmic reticulum in Arabidopsis. Cell 2013, 153, 562-574. [CrossRef] [PubMed]

16. Meng, Y.; Ma, X.; Chen, D.; Wu, P.; Chen, M. MicroRNA-mediated signaling involved in plant root development. Biochem. Biophys. Res. Commun. 2010, 393, 345-349. [CrossRef]

17. Meng, Y.; Wu, P.; Chen, M. MicroRNAs in plant roots: Current understanding and future perspectives. In Non Coding RNAs in Plants; Springer: Berlin/Heidelberg, Germany, 2011; pp. 269-284.

18. Guo, H.S.; Xie, Q.; Fei, J.F.; Chua, N.H. MicroRNA directs mRNA cleavage of the transcription factor NAC1 to downregulate auxin signals for Arabidopsis lateral root development. Plant Cell 2005, 17, 1376-1386. [CrossRef]

19. Mallory, A.C.; Bartel, D.P.; Bartel, B. MicroRNA-directed regulation of Arabidopsis AUXIN RESPONSE FACTOR17 is essential for proper development and modulates expression of early auxin response genes. Plant Cell 2005, 17, 1360-1375. [CrossRef]

20. Bustos-Sanmamed, P.; Mao, G.; Deng, Y.; Elouet, M.; Khan, G.A.; Bazin, J.; Turner, M.; Subramanian, S.; $\mathrm{Yu}, \mathrm{O}$; Crespi, M. Overexpression of miR160 affects root growth and nitrogen-fixing nodule number in Medicago truncatula. Funct. Plant Biol. 2013, 40, 1208-1220. [CrossRef] 
21. Marin, E.; Jouannet, V.; Herz, A.; Lokerse, A.S.; Weijers, D.; Vaucheret, H.; Nussaume, L.; Crespi, M.D.; Maizel, A. miR390, Arabidopsis TAS3 tasiRNAs, and their AUXIN RESPONSE FACTOR targets define an autoregulatory network quantitatively regulating lateral root growth. Plant Cell 2010, 22, 1104-1117. [CrossRef]

22. Tsikou, D.; Yan, Z.; Holt, D.B.; Abel, N.B.; Reid, D.E.; Madsen, L.H.; Bhasin, H.; Sexauer, M.; Stougaard, J.; Markmann, K. Systemic control of legume susceptibility to rhizobial infection by a mobile microRNA. Science 2018, 362, 233-236. [CrossRef]

23. Snyman, M.C.; Solofoharivelo, M.-C.; Souza-Richards, R.; Stephan, D.; Murray, S.; Burger, J.T. The use of high-throughput small RNA sequencing reveals differentially expressed microRNAs in response to aster yellows phytoplasma-infection in Vitis vinifera cv. 'Chardonnay'. PLoS ONE 2017, 12, e0182629. [CrossRef]

24. Sun, X.; Fan, G.; Su, L.; Wang, W.; Liang, Z.; Li, S.; Xin, H. Identification of cold-inducible microRNAs in grapevine. Front. Plant Sci. 2015, 6, 595. [CrossRef] [PubMed]

25. Kullan, J.B.; Pinto, D.L.P.; Bertolini, E.; Fasoli, M.; Zenoni, S.; Tornielli, G.B.; Pezzotti, M.; Meyers, B.C.; Farina, L.; Pè, M.E. miRVine: A microRNA expression atlas of grapevine based on small RNA sequencing. BMC Genom. 2015, 16, 393.

26. Mica, E.; Piccolo, V.; Delledonne, M.; Ferrarini, A.; Pezzotti, M.; Casati, C.; Del Fabbro, C.; Valle, G.; Policriti, A.; Morgante, M. High throughput approaches reveal splicing of primary microRNA transcripts and tissue specific expression of mature microRNAs in Vitis vinifera. BMC Genom. 2009, 10, 558. [CrossRef] [PubMed]

27. Chitarra, W.; Pagliarani, C.; Abbà, S.; Boccacci, P.; Birello, G.; Rossi, M.; Palmano, S.; Marzachì, C.; Perrone, I.; Gambino, G. miRVIT: A novel miRNA database and its application to uncover vitis responses to flavescence dorée infection. Front. Plant Sci. 2018, 9, 1034. [CrossRef] [PubMed]

28. Wang, J.-W.; Wang, L.-J.; Mao, Y.-B.; Cai, W.-J.; Xue, H.-W.; Chen, X.-Y. Control of root cap formation by microRNA-targeted auxin response factors in Arabidopsis. Plant Cell 2005, 17, 2204-2216. [CrossRef]

29. Chen, Q.; Deng, B.; Gao, J.; Zhao, Z.; Chen, Z.; Song, S.; Wang, L.; Zhao, L.; Xu, W.; Zhang, C. Comparative Analysis of miRNA Abundance Revealed the Function of Vvi-miR828 in Fruit Coloring in Root Restriction Cultivation Grapevine (Vitis vinifera L.). Int. J. Mol. Sci. 2019, 20, 4058. [CrossRef]

30. Yu, N.; Niu, Q.W.; Ng, K.H.; Chua, N.H. The role of miR156/SPLs modules in Arabidopsis lateral root development. Plant J. 2015, 83, 673-685. [CrossRef]

31. Bao, M.; Bian, H.; Zha, Y.; Li, F.; Sun, Y.; Bai, B.; Chen, Z.; Wang, J.; Zhu, M.; Han, N. miR396a-mediated basic helix-loop-helix transcription factor bHLH74 repression acts as a regulator for root growth in Arabidopsis seedlings. Plant Cell Physiol. 2014, 55, 1343-1353. [CrossRef]

32. Huang, W.; Peng, S.; Xian, Z.; Lin, D.; Hu, G.; Yang, L.; Ren, M.; Li, Z. Overexpression of a tomato miR171 target gene SIGRAS 24 impacts multiple agronomical traits via regulating gibberellin and auxin homeostasis. Plant Biotechnol. J. 2017, 15, 472-488. [CrossRef]

33. Gutierrez, L.; Bussell, J.D.; Pacurar, D.I.; Schwambach, J.; Pacurar, M.; Bellini, C. Phenotypic Plasticity of Adventitious Rooting in Arabidopsis is Controlled by Complex Regulation of AUXIN RESPONSE FACTOR Transcripts and MicroRNA Abundance. Plant Cell 2009, 21, 3119-3132. [CrossRef]

34. Zhu, Q.-H.; Fan, L.; Liu, Y.; Xu, H.; Llewellyn, D.; Wilson, I. miR482 regulation of NBS-LRR defense genes during fungal pathogen infection in cotton. PLoS ONE 2013, 8, e84390. [CrossRef] [PubMed]

35. González, V.M.; Müller, S.; Baulcombe, D.; Puigdomènech, P. Evolution of NBS-LRR gene copies among dicot plants and its regulation by members of the miR482/2118 superfamily of miRNAs. Mol. Plant 2015, 8, 329-331. [CrossRef] [PubMed]

36. Wang, W.; Galili, G. Tuning the Orchestra: miRNAs in Plant Immunity. Trends Plant Sci. 2019, 24, $189-191$. [CrossRef] [PubMed]

37. Kinoshita, N.; Wang, H.; Kasahara, H.; Liu, J.; MacPherson, C.; Machida, Y.; Kamiya, Y.; Hannah, M.A.; Chua, N.-H. IAA-Ala Resistant3, an evolutionarily conserved target of miR167, mediates Arabidopsis root architecture changes during high osmotic stress. Plant Cell 2012, 24, 3590-3602. [CrossRef]

38. Ni, Z.; Hu, Z.; Jiang, Q.; Zhang, H. GmNFYA3, a target gene of miR169, is a positive regulator of plant tolerance to drought stress. Plant Mol. Biol. 2013, 82, 113-129. [CrossRef]

39. Bakhshi, B.; Fard, E.M.; Hosseini, G. Evaluation of miR398 differential expression in rice under drought stress condition. Bull. Georg. Natl. Acad. Sci 2014, 8, 87-92. 
40. Ma, C.; Burd, S.; Lers, A. miR408 is involved in abiotic stress responses in Arabidopsis. Plant J. 2015, 84, 169-187. [CrossRef]

41. Martin, M. Cutadapt removes adapter sequences from high-throughput sequencing reads. EMBnet. J. 2011, 17, 10-12. [CrossRef]

42. Gordon, A.; Hannon, G. Fastx-toolkit. FASTQ/A Short-Reads Preprocessing Tools. Unpublished. May 2010. Available online: http://hannonlab.cshl.edu/fastx_toolkit (accessed on 5 April 2020).

43. Patel, R.K.; Jain, M. NGS QC Toolkit: A toolkit for quality control of next generation sequencing data. PLOS ONE 2012, 7, e30619. [CrossRef]

44. Griffiths-Jones, S.; Bateman, A.; Marshall, M.; Khanna, A.; Eddy, S.R. Rfam: An RNA family database. Nucleic Acids Res. 2003, 31, 439-441. [CrossRef]

45. Altschul, S.F.; Gish, W.; Miller, W.; Myers, E.W.; Lipman, D.J. Basic local alignment search tool. J. Mol. Biol. 1990, 215, 403-410. [CrossRef]

46. Langmead, B.; Salzberg, S.L. Fast gapped-read alignment with Bowtie 2. Nat. Methods 2012, 9, 357. [CrossRef] [PubMed]

47. Tarailo-Graovac, M.; Chen, N. Using RepeatMasker to identify repetitive elements in genomic sequences. Curr. Protoc. Bioinform. 2009, 25, 4.10.1-4.10.14. [CrossRef] [PubMed]

48. Griffiths-Jones, S.; Saini, H.K.; van Dongen, S.; Enright, A.J. miRBase: Tools for microRNA genomics. Nucleic Acids Res. 2007, 36 (Suppl. 1), D154-D158. [CrossRef]

49. Denman, R.B. Using RNAFOLD to predict the activity of small catalytic RNAs. Biotechniques 1993, 15, 1090-1095. [PubMed]

50. Sun, J.; Wang, S.; Li, C.; Ren, Y.; Wang, J. Novel expression profiles of microRNAs suggest that specific miRNAs regulate gene expression for the sexual maturation of female Schistosoma japonicum after pairing. Parasites Vectors 2014, 7, 177. [CrossRef] [PubMed]

51. Anders, S. Analysing RNA-Seq data with the DESeq package. Mol. Biol. 2010, 43, 1-17.

52. Fahlgren, N.; Carrington, J.C. miRNA target prediction in plants. In Plant MicroRNAs; Springer: Berlin/Heidelberg, Germany, 2010; pp. 51-57.

53. Hall, T.; Biosciences, I.; Carlsbad, C. BioEdit: An important software for molecular biology. GERF Bull. Biosci. 2011, 2, 60-61.

54. Tamura, K.; Stecher, G.; Peterson, D.; Filipski, A.; Kumar, S. MEGA6: Molecular evolutionary genetics analysis version 6.0. Mol. Biol. Evol. 2013, 30, 2725-2729. [CrossRef]

55. Luo, M.; Gao, Z.; Li, H.; Li, Q.; Zhang, C.; Xu, W.; Song, S.; Ma, C.; Wang, S. Selection of reference genes for miRNA qRT-PCR under abiotic stress in grapevine. Sci. Rep. 2018, 8, 4444. [CrossRef] 